\title{
Multi-omic Characterization of the Taxa-function Relationship in Infant Gut Microbiomes
}

\section{Quang P Nguyen}

Dartmouth College https://orcid.org/0000-0002-2072-3279

Margaret R. Karagas

Dartmouth College

Juliette C. Madan

Dartmouth College Geisel School of Medicine

\section{Erika Dade}

Dartmouth College

Tom J. Palys

Dartmouth College

Hilary G. Morrison

Marine Biological Laboratory

Wimal W. Pathmasiri

UNC-Chapel Hill: University of North Carolina at Chapel Hill

\section{Susan McRitche}

UNC-Chapel Hill: University of North Carolina at Chapel Hill

\section{Susan J. Sumner}

UNC-Chapel Hill: University of North Carolina at Chapel Hill

\section{H. Robert Frost}

Dartmouth College

Anne G. Hoen ( $\nabla$ Anne.G.Hoen@dartmouth.edu )

Dartmouth College

\section{Research}

Keywords: Infant gut microbiome, stool metabolome, prediction models, functional redundancy, metabolism

Posted Date: September 22nd, 2020

DOI: https://doi.org/10.21203/rs.3.rs-79308/v1

License: (9) This work is licensed under a Creative Commons Attribution 4.0 International License. 

1 Multi-omic characterization of the taxa-function relationship in infant gut microbiomes

2 Quang P. Nguyen ${ }^{1,2}$, Margaret R. Karagas ${ }^{1,3}$, Juliette C. Madan ${ }^{1,2,3,4}$, Erika Dade ${ }^{1}$, Tom J. Palys ${ }^{1}$, Hilary G.

3 Morrison $^{5}$, Wimal W. Pathmasiri $6^{*}$, Susan McRitche ${ }^{6}$, Susan J. Sumner ${ }^{6}$, H. Robert Frost ${ }^{2^{* *}}$ \& Anne G. Hoen ${ }^{1,2,3^{* *}}$

$4 \quad{ }^{1}$ Department of Epidemiology, Geisel School of Medicine at Dartmouth College, Hanover, NH, USA.

$5 \quad{ }^{2}$ Department of Biomedical Data Science, Geisel School of Medicine at Dartmouth College, Hanover, NH, USA

$6 \quad{ }^{3}$ Children's Environmental Health \& Disease Prevention Research Center at Dartmouth, Lebanon, NH, USA.

$7 \quad{ }^{4}$ Division of Neonatology, Department of Pediatrics, Children's Hospital at Dartmouth, Hanover, NH, USA.



$9{ }^{6}$ Department of Nutrition, Nutrition Research Institute, University of North Carolina at Chapel Hill, Chapel Hill, 10 NC, USA

11 Corresponding author: anne.g.hoen@dartmouth.edu

12 *Corresponding author for metabolomic assays: wimal pathmasiri@unc.edu

$13 * *$ Jointly supervised this research

\section{Email Addresses:}

15 QPN: Quang.P.Nguyen.GR@dartmouth.edu

16 MRK: Margaret.R.Karagas@dartmouth.edu

17 JCM: Juliette.Madan@dartmouth.edu

18 ED: Erika.F.Dade@dartmouth.edu

19 TJP: Thomas.J.Palys@dartmouth.edu

20 HGM: morrison@mbl.edu

21 WWP: wimal pathmasiri@unc.edu 
SLM: susan mcritchie@unc.edu

23 SJS: susan sumner@unc.edu

24 HRF: hildreth.r.frost@dartmouth.edu

25 AGH: Anne.G.Hoen@dartmouth.edu

\section{Abstract}

28 Background: The infant intestinal microbiome plays an important role in metabolism and immune

29 development with impacts on lifelong health. The linkage between the taxonomic composition of the

30 microbiome and its metabolic phenotype is undefined and complicated by redundancies in the taxon-function

31 relationships within microbial communities. To inform a more mechanistic understanding of the relationship between the microbiome and health, we performed an integrative statistical and machine learning-based analysis of microbe taxonomic structure and metabolic function in order to characterize the taxa-function relationship in early life.

Results: Stool samples collected from infants enrolled in the New Hampshire Birth Cohort Study (NHBCS) at approximately 6-weeks $(n=168)$ and 12-months $(n=282)$ of age were profiled using targeted and untargeted nuclear magnetic resonance (NMR) spectroscopy as well as DNA sequencing of the V4-V5 hypervariable region from the bacterial 16S rRNA gene. There was significant inter-omic concordance based on Procrustes analysis of sample distances ( 6 weeks: $p=0.056 ; 12$ months: $p=0.001$ ), however this association was no longer significant when accounting for phylogenetic relationships using generalized UniFrac distance metric (6 weeks: $p=0.376 ; 12$ months: $p=0.069$ ). Sparse canonical correlation analysis showed significant correlation, as well as identifying sets of microbe/metabolites driving microbiome-metabolome relatedness. Performance of machine learning models varied across different metabolites, with support vector machines (radial basis 4 $4 !$ function kernel) being the consistently top ranked model. However, predictive $\mathrm{R}^{2}$ values demonstrated poor predictive performance across all models assessed (avg. $R^{2}:-5.06 \%--6$ weeks; $-3.7 \%--12$ months). 
Conversely, the Spearman correlation metric was higher (avg. correlation: $0.344-6$ weeks; $0.265-12$ months). This demonstrated that taxonomic relative abundance was not predictive of metabolite concentrations.

49 Conclusions: Our results suggest a degree of overall association between taxonomic profiles and metabolite concentrations. However, lack of predictive capacity for stool metabolic signatures reflects, in part, the possible

51 role of functional redundancy in defining the taxa-function relationship in early life as well as the bidirectional

52 nature of the microbiome-metabolome association. Our results provide evidence in favor of a multi-omic 53 approach for microbiome studies, especially those focused on health outcomes.

54 Keywords: Infant gut microbiome, stool metabolome, prediction models, functional redundancy, metabolism

\section{Background}

56 The human gut microbiome is a complex and diverse system of microorganisms that co-inhabit the intestinal lumen and play a crucial role in modulating human health and disease [1, 2]. The development of the microbiota in early life is a sensitive process akin to primary ecological succession [3], and therefore both reliant on and vulnerable to external perturbations. Studies have linked microbiome alterations to long-term health consequences, including risk of obesity [4], type I diabetes [5], and inflammatory bowel disease [6]. As such, there is a need to understand how the microbiome participates in the multifactorial pathways leading to long-term disease outcomes. One key to this open question lies in the currently undefined relationship between the taxonomic structure of the microbiome and its metabolic phenotype. Previous studies addressing this question have mainly focused on DNA-based profiling of microbial functional potential, which, due to complicated regulatory mechanisms at the cellular level beyond the genome, is not equivalent to the microbiota's realized functional landscape [7].

67 There exists a bidirectional association between the metabolome and the microbiome in the gut $[8,9]$. These

68 low molecular weight molecules can either be nutrients that shape the composition of the microbiome [10] or 69 important byproducts of host-microbe nutrient co-metabolism that help regulate host metabolic homeostasis 
[11-13]. For example, members of the Clostridium clusters can produce and increase serum levels of branched chain amino acids, which are markers for insulin resistance and diabetes [14, 15]. However, studies suggest that the fecal metabolome specifically can be used as a readout of gut microbe metabolic functions. Zierer et al. [16] showed in a large cohort of adult females $(n=786)$ from the TwinsUK study that around $60 \%$ of the fecal metabolome is associated with microbial composition, where on average, $67 \%$ of variance in the metabolome can be explained by the microbiome.

76 Recent studies have integrated the metabolome in microbiome analyses of health outcomes, most notably Lloyd et al. [17] from the integrative Human Microbiome Project. However, these studies have mostly focused on adult populations with specific metabolic disease etiologies such as inflammatory bowel disease. Only a 79 limited number of studies [18-23] have simultaneously profiled the gut microbiome and metabolome from 80 infant stool samples. These studies have preliminarily established that metabolomic profiles shift as taxonomic 81 abundances change between subject case/control status [18, 20, 21, 24]. Specifically, Ayeni et al. $(n=48)$ [19] and Kisuse et al. $(n=35)$ [23] demonstrated that inter-sample distances calculated using metabolite abundances are correlated with those calculated from taxonomic profiles using Mantel tests across African and Asian cohorts. However, studies to date have either focused on preterm infants $[18,20,21]$ or had small sample sizes (less than 50) [19, 22, 23]. We identified a major gap in defining microbiome-metabolome relatedness among infants from a population-based cohort capturing both early in infancy and near the first birthday, with regards to determining the strength of association and to identify key contributors to the overall concordance.

Here, we present our study investigating associations between microbe abundances assayed with 16S rRNA sequencing and metabolomic profiles measured with ${ }^{1} \mathrm{H}$ NMR spectroscopy in a cohort of infants representing a rural general population from the New Hampshire Birth Cohort Study (NHBCS). This is a unique epidemiological cohort with multi-omic profiling of infant stool samples at multiple time points accompanied with rich sociodemographic, dietary and health outcomes data [25]. Our study utilizes predictive modeling, 
94 multivariate correlation methods and distance-based approaches to characterize the dynamic relationship 95 between the gut microbiome and the gut metabolome in early life.

\section{Results}

97 The overall workflow and subject selection process are described in Figure 1. Primary analyses were 98 performed on paired microbiome-metabolome data sets on samples collected at approximately 6 weeks $(\mathrm{N}=$ 158 ) and 12 months $(\mathrm{N}=282)$ of age (65 subjects have paired samples collected at both time points). In order to take advantage of the most samples from this study, each time point was analyzed separately with sensitivity analyses performed on sample pairs. After processing and filtering, we evaluated a final taxonomic dataset of 48 genera in 6 weeks samples and 72 genera in 12 months samples. Metabolomic profiles were represented as 208 unique untargeted spectral bins and a concentration-fitting method [26] was used to acquire specific relative concentrations of 36 targeted metabolites. These metabolites were chosen based on a literature search (Table S1) for compounds known to be associated with commensal gut microbes. Results presented here will primarily feature the targeted dataset, with accompanying figures and tables for the untargeted data set in the supplemental section. Figure 1 shows the overall workflow including the sample selection process.

\section{Study population}

Study subject characteristics are summarized in Table 1 separately for both subjects providing samples at 6week of age $(n=158)$ and 12-months of age $(n=282)$. Characteristic of our population, most infants are White (97.5\% among subjects contributing a 6-week sample; 95.4\% among subjects contributing a 12-month sample), delivered vaginally (6 weeks samples: 72.2\%; 12 months samples: $70.9 \%$ ) and did not have any systemic antibiotic exposure during initial hospitalization following birth (6 weeks samples: $95.6 \%$; 12 months samples $97.2 \%$ ). The average birth weight was also similar across subjects irrespective of the sample time point, $3370 \mathrm{~g}( \pm 480)$ for infants contributing 6-week samples and $3430 \mathrm{~g}( \pm 528)$ for infants contributing 12month samples. Similarly, the average gestational age was 39.1 weeks ( \pm 1.59$)$ (6-week samples) and 39 weeks ( \pm 1.7 ) (12-month samples). At the time of 6 -week sample collection, $62 \%$ of infants had been 
exclusively breastfed while by the time of 12 -month sample collection, $59.2 \%$ of infants received breast milk

120 supplemented with formula, however a large minority (35.1\%) remained exclusively breastfed. There were 121 more male than female infants in the cohort (53.8\% male among infants contributing a 6-week sample; $56.4 \%$ 122 male among infants contributing a 12-month sample). Maternal smoking during pregnancy was rare (6-week 123 samples: 7\%; 12-month samples: $5 \%$ ).

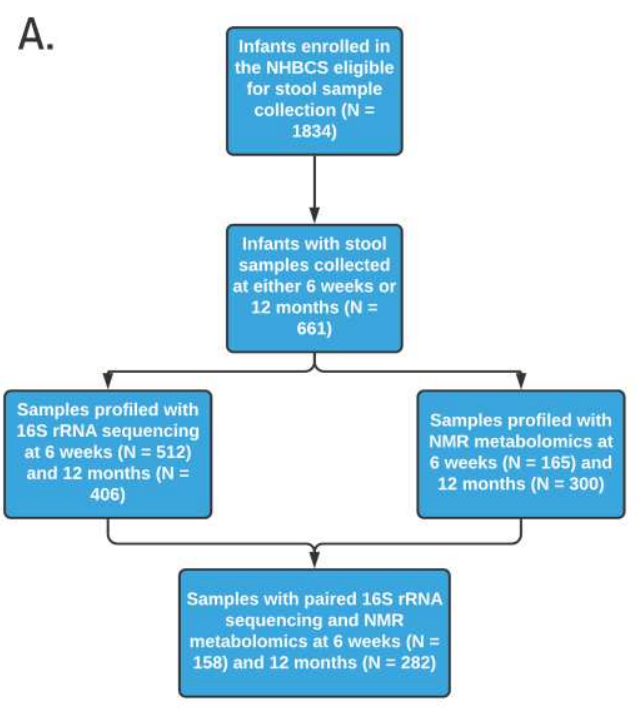

B.

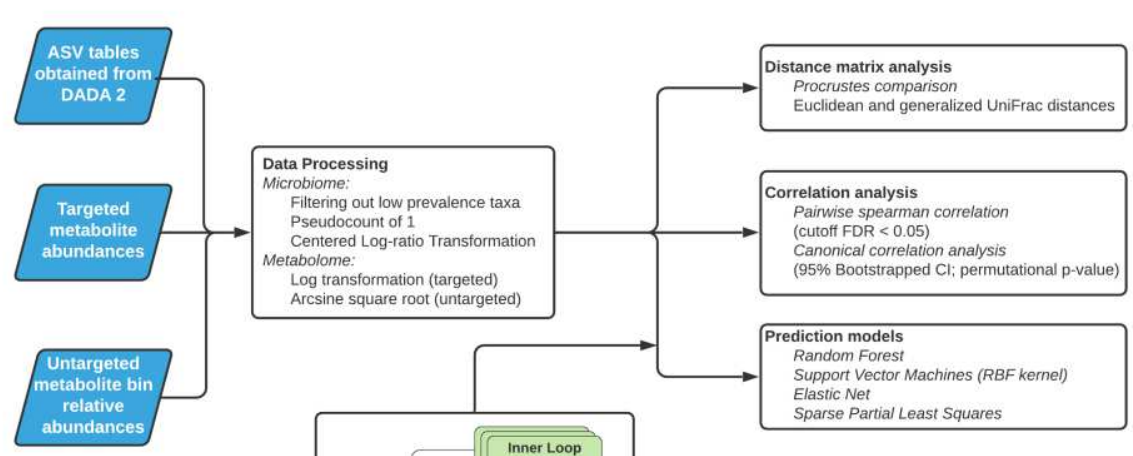

Figure 1. Overview of the analysis. Panel A describes the subject selection workflow and panel B describes the analytic pipeline.

Table 1. Selected characteristics of subjects contributing samples at 6 weeks $(n=158)$ and at 12 months of age $(n=282)$.

\section{6 weeks \\ $(n=158)$}

12 months

$(n=282)$

\section{Birthweight (grams)}

Mean (Standard Deviation)

Median [Minimum, Maximum]
3370 (480)

$3430[1910,4710]$
3430 (528)

3450 [1320, 4660] 


\begin{tabular}{|c|c|c|}
\hline Missing & $2(1.3 \%)$ & $4(1.4 \%)$ \\
\hline \multicolumn{3}{|l|}{ Sex } \\
\hline Male & $85(53.8 \%)$ & $159(56.4 \%)$ \\
\hline Female & $73(46.2 \%)$ & $123(43.6 \%)$ \\
\hline \multicolumn{3}{|c|}{ Feeding Mode Until Time of Sample } \\
\hline Unknown & $6(3.8 \%)$ & $7(2.5 \%)$ \\
\hline Exclusively breastfed & $98(62 \%)$ & $99(35.1 \%)$ \\
\hline Exclusively formula fed & $13(8.2 \%)$ & $9(3.2 \%)$ \\
\hline Mixed & $41(25.9 \%)$ & $167(59.2 \%)$ \\
\hline \multicolumn{3}{|l|}{ Delivery Mode } \\
\hline Vaginal & $114(72.2 \%)$ & $200(70.9 \%)$ \\
\hline Cesarean & $44(27.8 \%)$ & $82(29.1 \%)$ \\
\hline \multicolumn{3}{|l|}{ Gestational Age (Weeks) } \\
\hline Mean (SD) & $39.1(1.59)$ & $39.0(1.70)$ \\
\hline Median [Minimum, Maximum] & $39.1[33.4,43.0]$ & $39.1[29.1,42.0]$ \\
\hline $\begin{array}{l}\text { Post-delivery infant systemic } \\
\text { antibiotic exposure }\end{array}$ & & \\
\hline No & $151(95.6 \%)$ & $274(97.2 \%)$ \\
\hline Yes & $7(4.4 \%)$ & $8(2.8 \%)$ \\
\hline \multicolumn{3}{|c|}{ Maternal smoking during pregnancy } \\
\hline No & $143(90.5 \%)$ & $262(92.9 \%)$ \\
\hline Yes & $11(7.0 \%)$ & $14(5.0 \%)$ \\
\hline Missing & $4(2.5 \%)$ & $6(2.1 \%)$ \\
\hline Infant Race & & \\
\hline
\end{tabular}


Other

White
$4(2.5 \%)$

$154(97.5 \%)$
$13(4.6 \%)$

$269(95.4 \%)$

Inter-omic sample distance comparison suggests overall concordance between data sets

Global concordance between the microbiome and the metabolome was observed across both time points and metabolomic data sets (Figure 2A, Figure S1A) when analyzed using a symmetric Procrustes test with samples ordinated by Euclidean distances (Methods). It is noted that the p-value at 6 weeks for the targeted data set $(p=0.057)$ was only trending close to significant at the 0.05 level.

Since the Procrustes test was performed on principal coordinate (PCoA) ordinations of sample distances, the result is sensitive to the choice of dissimilarity metric. In addition to standard Euclidean distances, the generalized UniFrac (gUniFrac) metric was also leveraged to account for phylogeny in calculating differences between samples. With gUniFrac, the association was not significant at either time points for the targeted data set only (Figure $2 \mathrm{~B}$ ), while the untargeted data set still maintained strong concordance (6 weeks samples $-\mathrm{p}=$ $0.001 ; 12$ months samples $-p=0.006$; Figure S1B). 
A. Euclidean-Euclidean

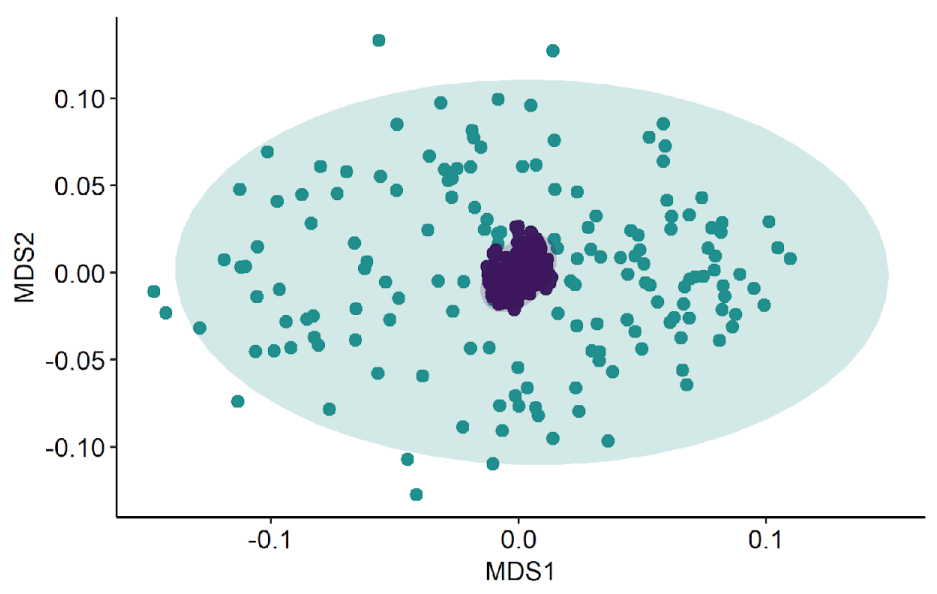

B. Gunifrac-Euclidean

$\mathrm{p}$-value $=0.376$

Sum of Squares: 0.99

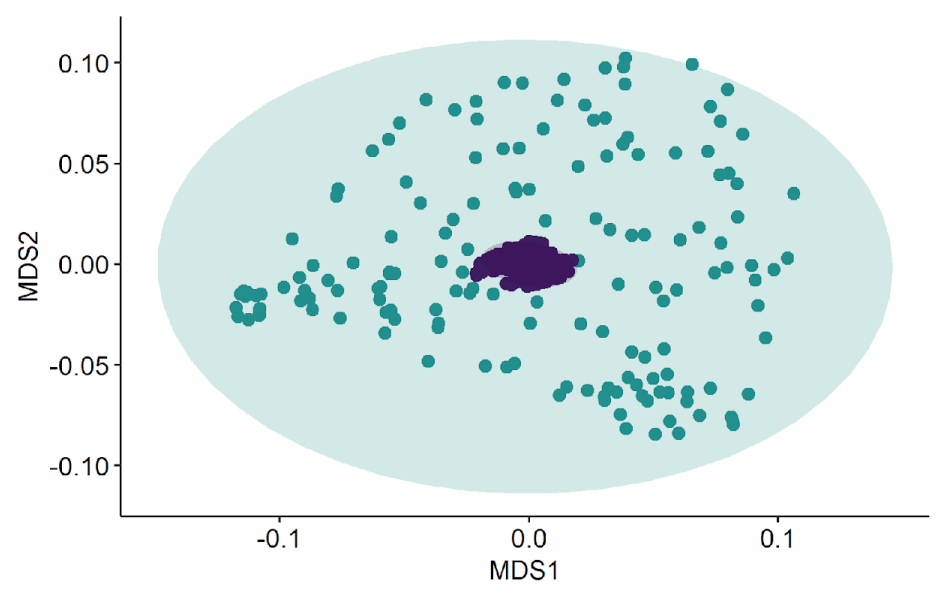

6 weeks

Sum of Squares: 0.98 p-value $=0.057$

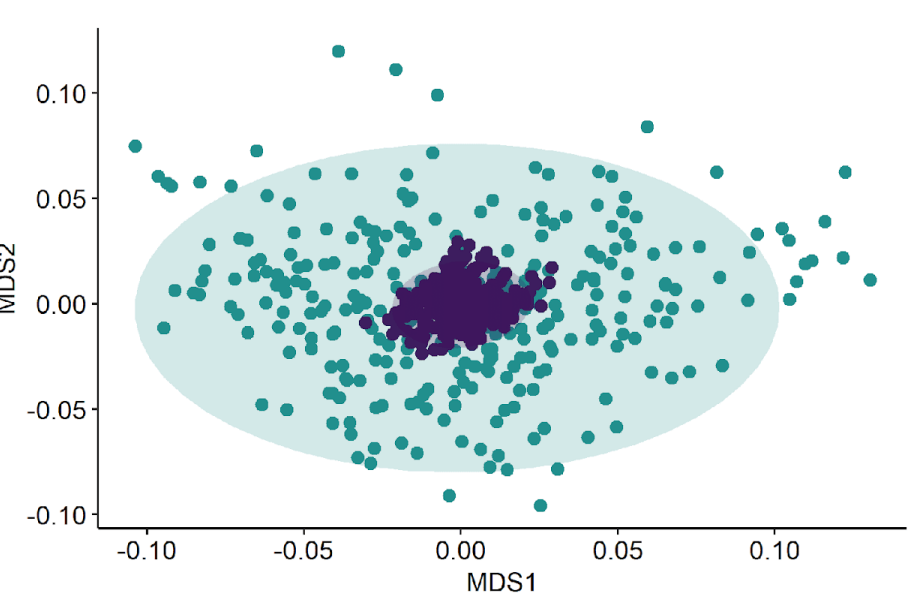

p-value $=0.069$

Sum of Squares: 0.99

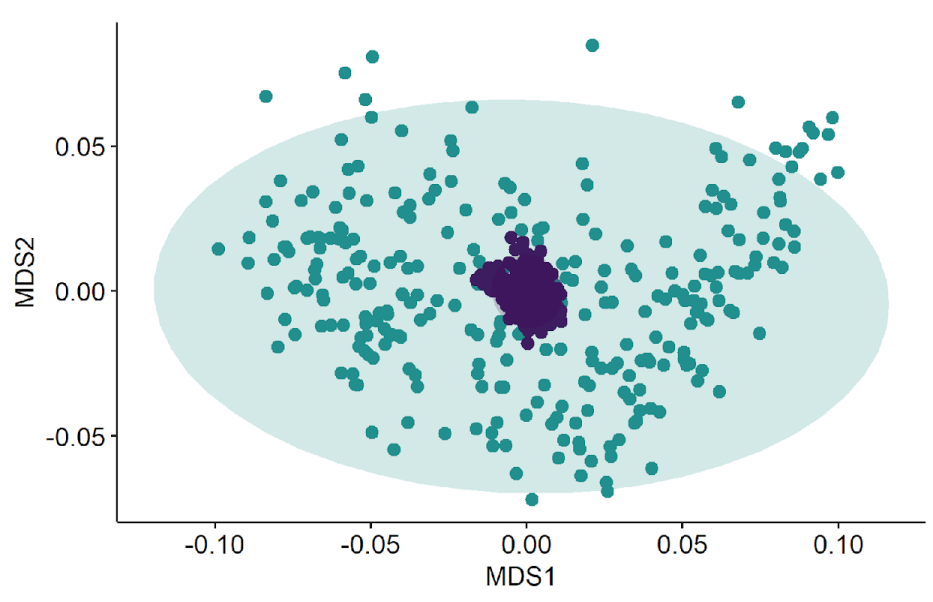

12 months

Figure 2: Inter-omics Procrustes biplots comparing PCoA ordinations of targeted metabolite profiles and

143 taxonomic relative abundances for 6 weeks (left panels) $(n=158)$ and 12 months (right panels) $(n=262)$. Top

144 panels present analyses based on ordinations from Euclidean distances of genus level abundances after centered log ratio transformation and Euclidean distances of log-transformed metabolite profiles. Bottom panel presents analyses based on gUniFrac distance of amplicon sequence variant (ASV) relative abundances and Euclidean distances of log-transformed metabolite profiles. There were significant associations between the 
microbiome and the metabolome (both targeted and untargeted) when utilizing Euclidean distances, however

149 this association goes away when the gUniFrac distance was employed for the targeted metabolites only.

\section{A. 6 Weeks}
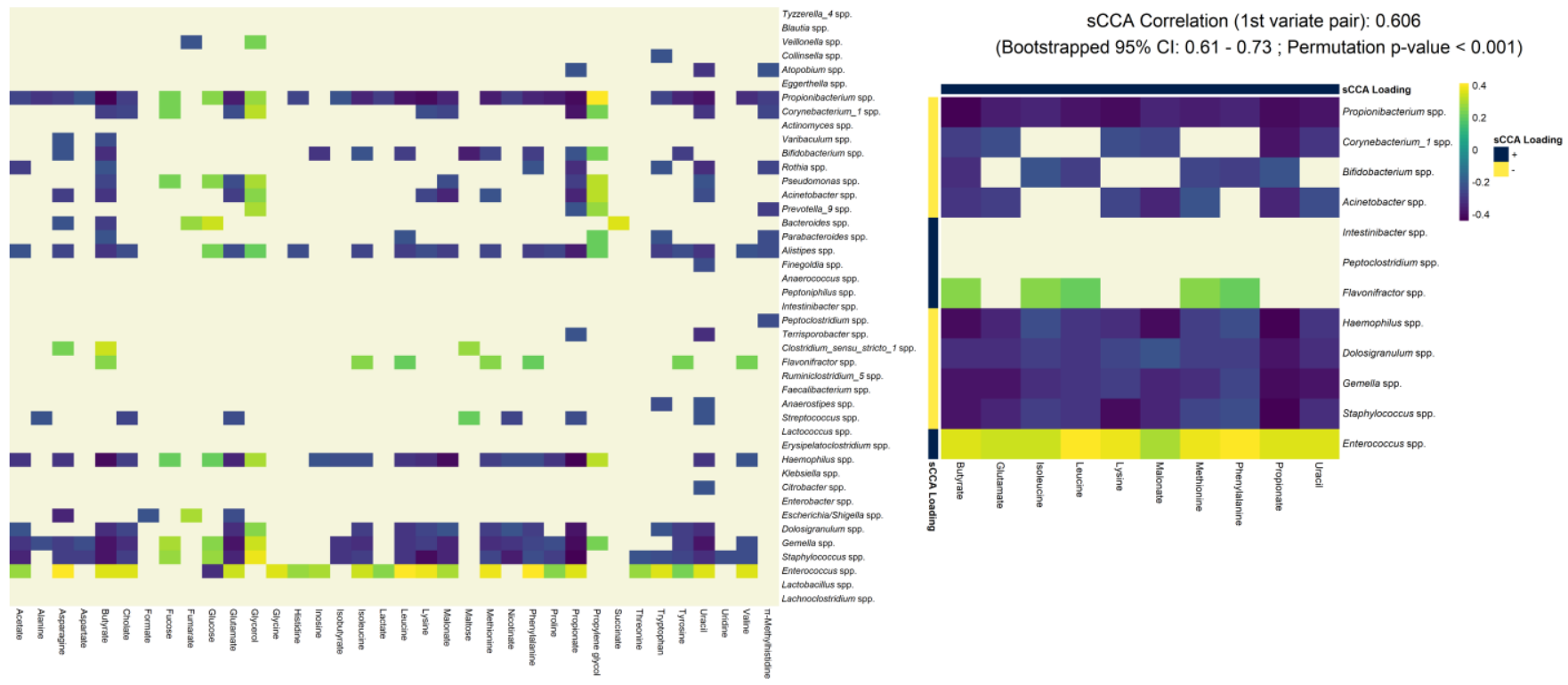

B. 12 Months
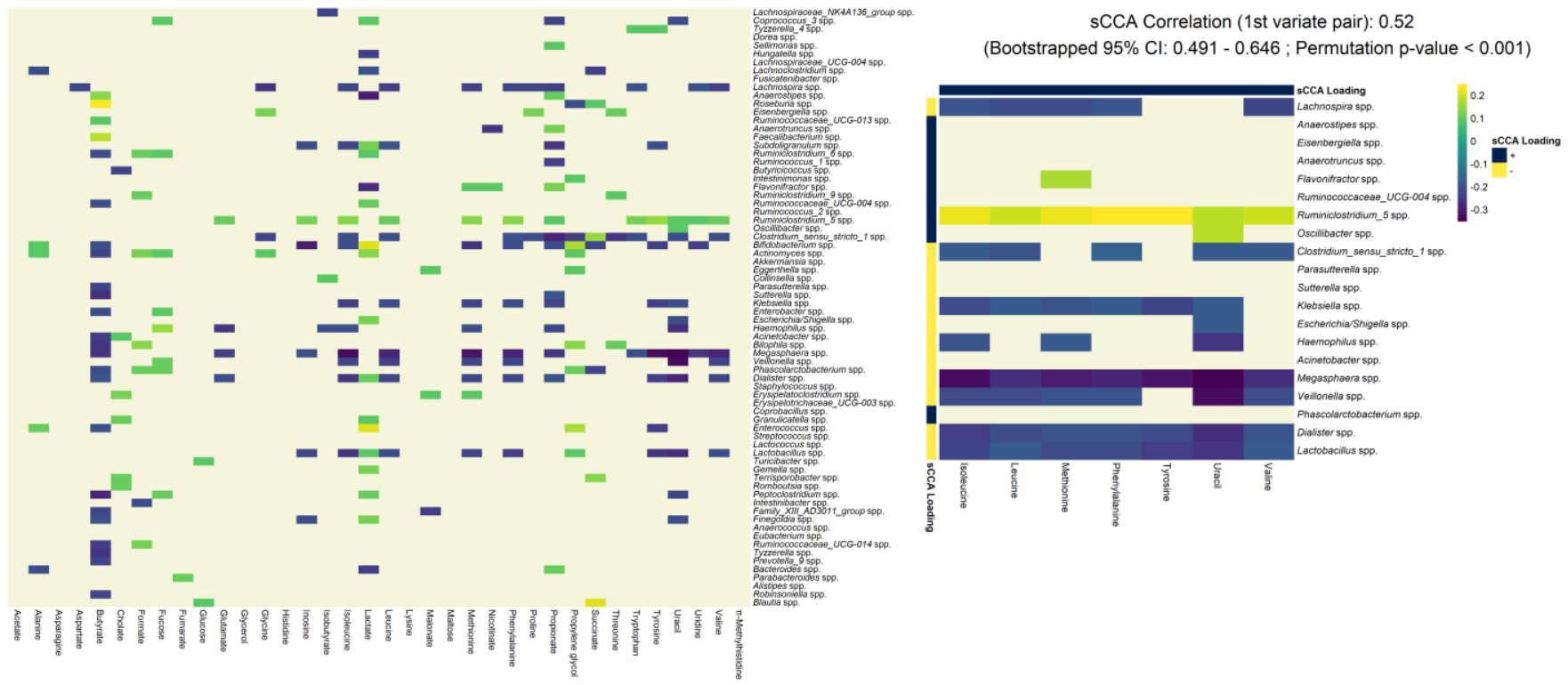

Figure 3: Pairwise Spearman correlation of concentration-fitted metabolites and genus-level taxonomic abundances for 6-weeks (panel $A, N=168$ ) and 12-months (panel $B, N=282$ ) infants. Left panel displays the overall correlation pattern, where non-significant correlations are not colored (false discovery rate (FDR) 
controlled q-value $<0.05)$. Right panel displays the same heatmap restricted to taxa and metabolites selected by the sparse CCA procedure. Additionally, correlation coefficient of the first SCCA variate pair, bootstrapped $95 \%$ confidence interval and permutation p-value are also reported. Significant microbiome-metabolome correlation was observed at both time points, however no significant difference was found between the time points.

\section{Sparse canonical correlation analyses reveal the core set of microbe-metabolite groups driving inter-} omic relatedness.

Given broad concordance between the gut microbiome and metabolome from sample distance analyses, we employed sparse canonical correlation analysis (SCCA) and pairwise Spearman rank correlation to ascertain the strength of association as well as to identify core microbes and metabolites driving this relationship (Methods).

The majority of taxa (65\% of total genera at 6 -weeks and $80 \%$ at 12 -months) and metabolites ( $100 \%$ of total metabolites at 6-weeks and $80 \%$ at 12-months) were part of significant (FDR threshold 0.05 ) Spearman pairwise comparisons (Supplementary Note 1). This demonstrated a high level of congruence, where most microbes are involved in metabolic processes captured in the stool metabolome. This was also reflected in the significant multivariate correlation (permutation p. < 0.001). However, at 6 weeks (correlation: 0.606 [0.61 $0.73]$ ), the degree of concordance was slightly higher than at 12 months (correlation: 0.52 [0.431 - 0.646]) but this difference was not significant due to overlapping confidence intervals. The canonical correlation was slightly higher in the untargeted data set (6 weeks: 0.636 [0.621 - 0.733]; 12 months: 0.49 [0.475 - 0.702]), however the difference between time points was similar (Figure S2, Supplemental Note 2).

Using SCCA, we identified a core set of microbes and metabolites that are major contributors to the multivariate correlation (Figure 3, right panels; Supplementary Notes 2). Selected microbes (in both the targeted and untargeted data set) belonged to the Firmicutes, Actinobacteria and Proteobacteria phyla, as those are the most commonly found phyla in the infant gut [25, 27]. However, previously established dominant genera such as Bifidobacterium, Bacteroides and Lactobacillus were not consistently selected across both time 
points. In the targeted data set Bifidobacterium was selected only at 6 weeks and Lactobacillus was only selected at 12 months. Most notably, more microbes were selected at 12 months compared to 6 weeks in the targeted data set, however in the untargeted data set this pattern was reversed (Figure S2, right panels). In terms of selected metabolites, the majority of the selected metabolites in the targeted data set were amino acids (Supplementary Table 1), with some short chain fatty acids (SCFAs) selected at the 6-week time point.

\section{Microbial community structure is weakly predictive of stool metabolite relative concentrations}

In order to determine how well the fecal metabolome acts as a functional representation of the gut microbiome, we fitted metabolite-specific prediction models based on taxonomic profiles. Chosen models include random forest (RF), elastic net (EN), support vector machines with radial basis kernel (SVM-RBF) and sparse partial least squares (SPLS), all of which had previously been shown to work well with microbiome-associated learning tasks [28]. Evaluation was based on predicted R-squared $\left(R^{2}\right)$ and Spearman correlation coefficient (SCC) as measured using 100 repeats of 5 -fold nested cross validation (Methods).

Predictive performance was more dependent on the metabolite being predicted than by choice of model (Figure 4, Supplementary Notes 3, Supplementary Files 1). Looking at predictive $\mathrm{R}^{2}$ (Figure 4 panel A), the average posterior mean performance across all models and metabolites was negative for both time points ($5.6 \%$ at 6 weeks; $-3.07 \%$ at 12 months), which indicated that for most prediction tasks the fitted model was less predictive than a naïve, intercept only model. At 6 weeks $22.2 \%$ of metabolites had models that perform significantly better than the null (lower bound of $95 \%$ credible interval larger than 0 ) while at 12 months $38.9 \%$ of metabolites fit the classification. However, even among such metabolites, the maximum $\mathrm{R}^{2}$ is only $11.8 \%$ at 6 weeks and $8.7 \%$ at 12 months. Conversely, SCC values were higher in comparison (cross-metabolite avg.: 0.339 at 6 weeks and 0.249 at 12 months) (Figure 4 panel B, Supplementary Notes 3). At 6 weeks, $83 \%$ of metabolites were significantly more performant than the null, while at 12 months all metabolites were selected. Using a more stringent cutoff as used by Mallick et al. [29], the majority of metabolites at 6 weeks $(69.4 \%$ of total metabolites) still remained as well predicted while conversely at 12 months only $38.9 \%$ (of total metabolites) were predictable. 
204

205

206

207

208

209

210

211

212

213

214

215

216

217

218

219

220

221

222

223

224

225

226

227

228

Results from the untargeted analysis showed higher performance values for both evaluation metrics (Supplementary Note 3). Specifically, $56.7 \%$ of metabolites bins at 6 weeks and $42.7 \%$ of bins at 12 months had $R^{2}$ values significantly higher than 0 . However, under SCC, while $57 \%$ of metabolite bins at 6 weeks had SCC values significantly larger than 0.3 cutoff, only $28.8 \%$ of metabolite bins at 12 months fit this criterion. Despite better performance, the overall average values were still low, suggesting that across the entire metabolome few metabolites were highly predictable.

Despite weak predictive performance values, we were still interested in determining a model that stands out as the most appropriate for our prediction task. Aggregating performance across metabolites stratified by model for both evaluation metrics (Figure 5, top panel), it can be observed that the average performances were similar (Supplementary Notes 3), for which no semi-targeted analyses performed better on average than the naive model under $R^{2}$. This is further illustrated when model performance was aggregated by rank using Borda scores (Figure 5, bottom panel). A higher score indicated that a model was selected as the top choice more times than others, where an even score distribution across models corroborated the suggestion that no model was best across all prediction tasks. That said, SVM-RBF seemed to be the highest scoring model, particularly for the 6-week time point. The untargeted analysis also found similar results (Figure S3).

\section{Sensitivity analyses}

We performed both Procrustes and correlation analyses on a data set restricted to the 65 subjects with paired samples collected at both time points ( 6 weeks and 12 months). Each time point was analyzed separately as in our main analysis. In the targeted data set, significant Procrustes concordance was observed at 12 months ( $p$ value $=0.003)$ but not at 6 weeks ( $p$-value $=0.106)$. This association was no longer significant when considering taxonomic ordination using the gUniFrac distance metric (6 weeks). Surprisingly, in the untargeted data set, no association was observed across both time points and choice of distance metric (Figure S5, S6). In the canonical correlation analyses, significance was only observed in the targeted data set at 6 weeks only ( 6 weeks: permutation $p$-value $=0.044 ; 12$ months: permutation $p$-value $=0.388$ ). Even though most correlations were not significantly different from the permuted null, the canonical correlation coefficient is 
229 higher at 6 weeks compared to 12 months in both the targeted ( 6 weeks: 0.676 [0.661 - 0.765]; 12 months: 0.52 [0.484 - 0.663]), and untargeted (6 weeks: 0.703 [0.685 - 0.788]; 12 months: 0.444 [0.52 - 0.705]) data sets (Figure S7, S8).

Furthermore, to ascertain the uncertainty of model choice, we evaluated all selected modelling approaches with simulated data sets based on bootstrapped resampling of taxonomic relative abundances (Figure S4). For the first simulation scenario, models were assessed against generated metabolite concentrations under different degrees of model saturation (number of taxa associated with the outcome) and association strength (signal to noise ratio). As expected, model performance asymptotically reached perfect prediction with increasing signal strength and model saturation, which demonstrated that prediction models were able to capture predictive associations should they arise even in sparse microbiome data sets. Most notably, simulation performance differed more by signal-to-noise ratio than model saturation, which indicated that the strength of association plays a larger role in the observed weak predictive performance than the number of taxa involved. Surprisingly, we obtained very similar results to our real data values under our lowest simulation setting (model saturation $=5 \%$; signal-to-noise ratio 0.5 ). As such, it can be suggested that the lack of predictability is due to weak coupling rather than model choice. 
A. R-squared
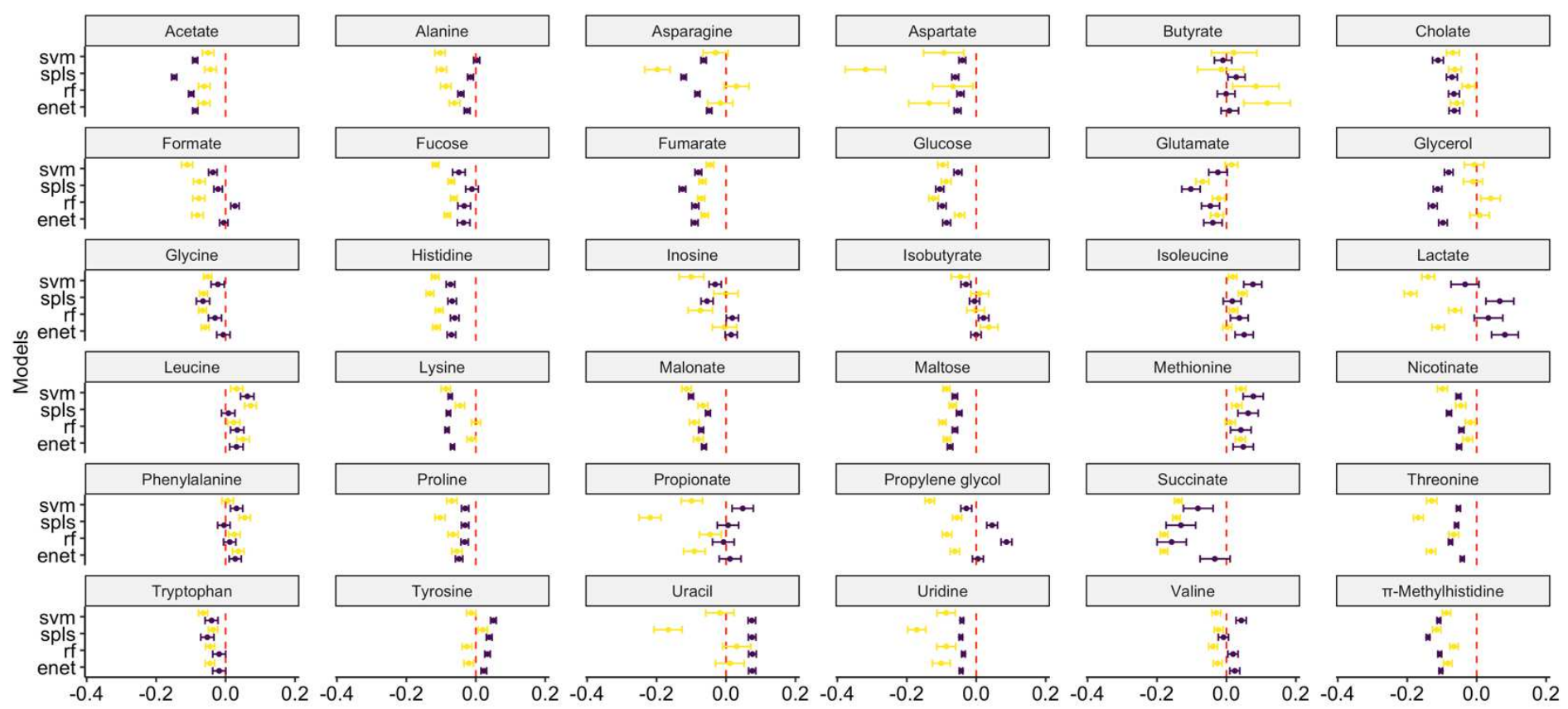

\section{B. Spearman Correlation}
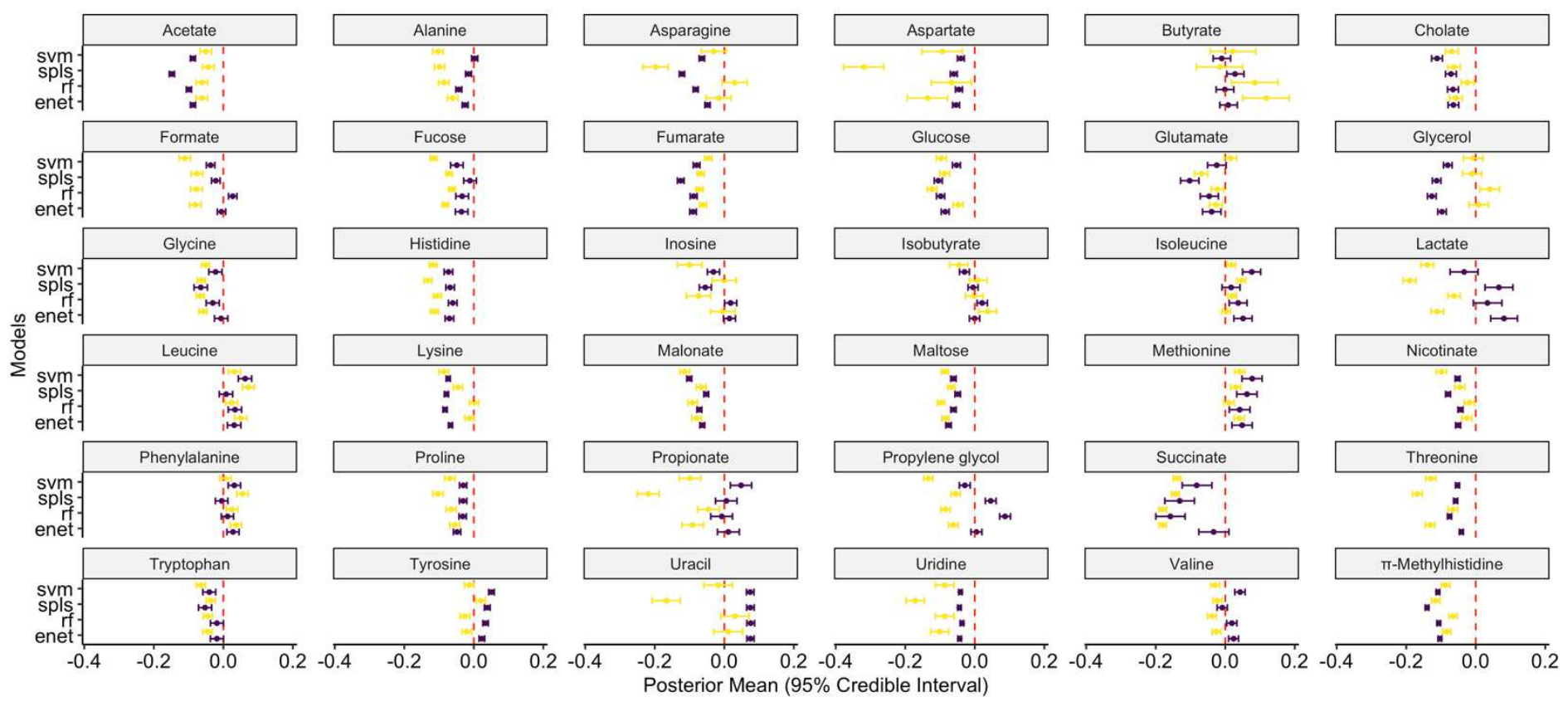

Figure 4. Forest plots of each prediction performance metric (R-squared - Panel A, Spearman correlation Panel B) for each time point ( 6 weeks $(n=158), 12$ months $(n=282))$ across all 36 metabolites and 4 machine learning models. 95\% credible interval and predictive posterior means were generated using Bayesian 
modelling of the evaluation statistic (Methods) after 100 repeats of 5 -fold nested cross validation. Red vertical

249 lines indicate a value of 0 for the evaluation metric (equivalent to null model). Metabolites were classified as

250 predictable if the null value did not lie within the estimated $95 \%$ credible interval. For most metabolites,

251 predictive performance was not significantly better than null models.
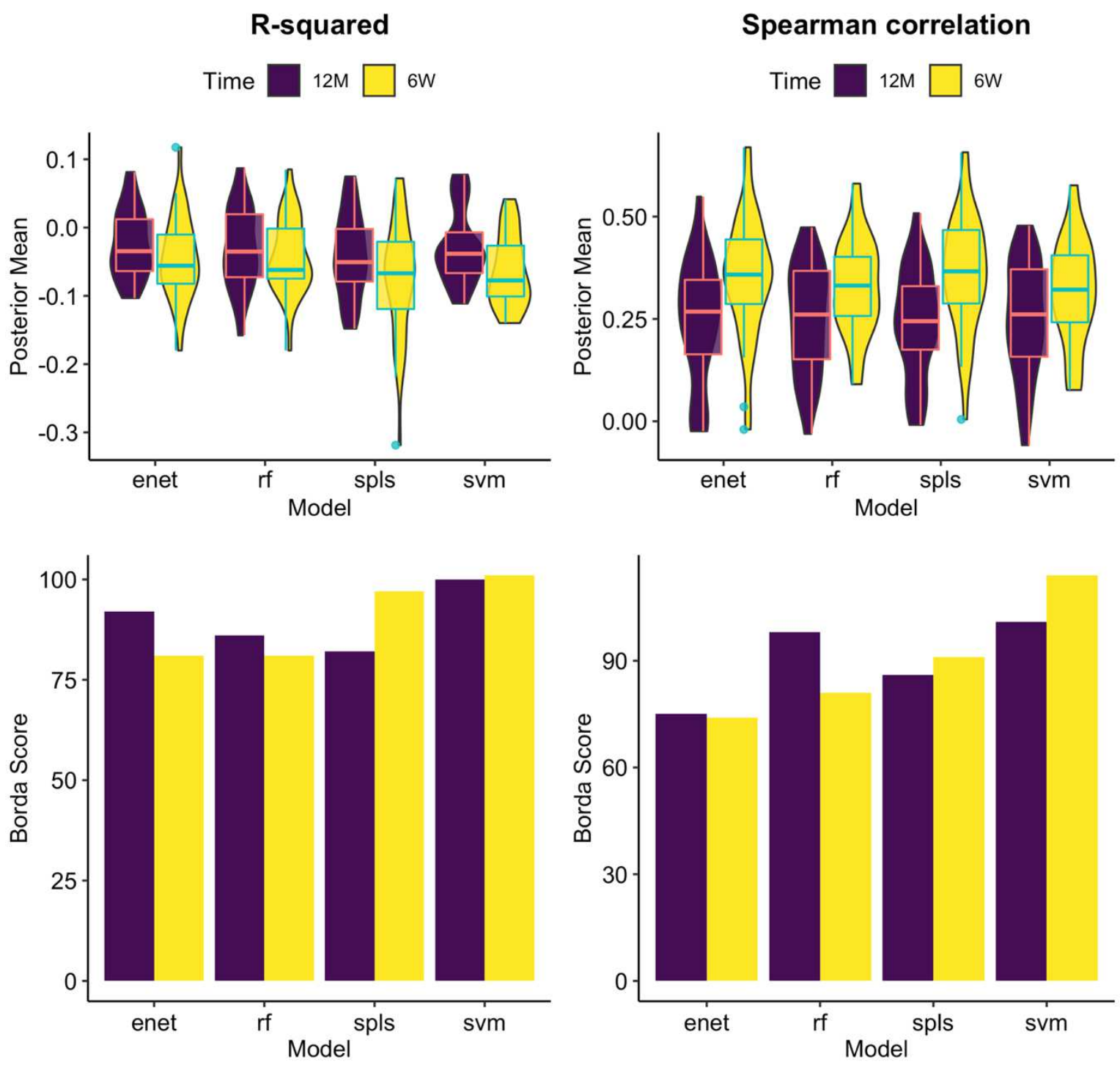

Figure 5. Comparative analysis predictive model performance across all metabolites in the targeted dataset for both 6-weeks $(n=158)$ and 12-months $(n=282)$ time points. Top panel shows superimposed boxplots and 
violin plots of the distribution of predictive posterior mean for each evaluation metric across all 36 metabolites. Bottom panels show aggregated model rankings for all metabolites using R-squared (left) and Spearman correlation (right) using Borda scores (Methods). Higher scores indicate that a model was consistently selected as a better performing. Relatively similar Borda scores and cross-metabolite average predictive performances indicate that no model was clearly the most performant. However, support vector machines (with radial basis function kernel) was highest scoring model.

\section{Discussion}

In this study, we analyzed the relationship between fecal microbial taxonomic abundances and metabolite concentrations with multi-omic profiling via paired targeted sequencing of the $16 \mathrm{~S}$ rRNA gene and $\mathrm{H}^{1} \mathrm{NMR}$ metabolomics at multiple time points. Ecological, statistical and machine learning approaches were applied to provide a multi-faceted view of this association. To our knowledge, this study is one of the few comprehensive investigations addressing the microbiome/metabolome interface in a large general population cohort of infants.

\section{The microbiome is significantly correlated but weakly predictive of the metabolome}

Overall global concordance was found from three independent methods (Procrustes analysis, SCCA and univariate Spearman correlation), consistent with previous studies on both infant $[19,24]$ and adult populations $[17,30]$. This overall effect was found at both time points, suggesting there coupling exists throughout infancy even when there is a high inter- and intra-individual variability in taxonomic compositions [27].

Although the microbiome and the metabolome were significantly correlated in our analyses, most metabolites were not predictable across chosen machine learning models when evaluated by $\mathrm{R}^{2}$ statistic. Even among the small number of metabolites that are significantly predictable compared to the null model, the maximum performance values were still low for both the untargeted and targeted analyses. Since all fitted models had been shown to be suited for microbiome-associated prediction tasks [28, 31] as well as covering both nonlinear and linear associations, the lack of predictability is unlikely due to model choice. Our data driven simulations further supported this assessment, where low predictability by $R^{2}$ was observed when there is low 
280

281

282

283

284

285

286

287

288

289

290

291

292

293

294

295

296

297

298

299

300

301

302

303

304

signal-to-noise ratio. However, when evaluated using SCC, the models seem to perform better. Most of the metabolome (57\% of metabolite bins) were significantly well predicted at SCC cut off criteria of 0.3 . This result is similar to Mallick et al. [29], which also performs microbiome to metabolome prediction using data from a cohort of adults. The study reported $53.8 \%$ of metabolites classified as well-predicted under the elastic net model with the same cutoff. Despite similar percentages of the metabolome being well predicted, the maximum cross-model average correlation value is 0.647 at 6 weeks and 0.431 at 12 months, which is lower than those seen in Mallick et al. Low $\mathrm{R}^{2}$ compared to SCC demonstrated that even though models were able to anticipate to a certain degree the trends in metabolite abundances, they were not able to accurately predict the exact concentrations.

Even though previous research suggests that the fecal metabolome is a functional representation of the gut microbiome [16], metabolites are often resultant of both host and microbial processes [11, 13]. This means for each metabolite, there are host-associated components that drive measured concentrations [32]. We hypothesize that metabolites that are more predictable are those which are associated with microbe-specific metabolism. As such, predictability can be a marker to identify whether a metabolite is host or microbiome driven.

However, poor overall performance even amongst predictable metabolites suggests the possible role of other ecological processes. We hypothesized functional redundancy, an aspect ubiquitous in microbial communities [33], plays an important role in this weak coupling. Under functional redundancy, high variability in taxonomic profiles would correspond to little or no change in metabolite abundances, making it difficult to form accurate predictions. Functional redundancy is usually a marker for ecosystem resilience [34]. This is evidenced when Procrustes analyses were adjusted for phylogenetic relatedness using the gUniFrac distance metric where inter-omic association in the targeted data set is no longer significant compared to using Euclidean distances. gUniFrac adjusts for phylogeny by weighting the differences in proportions of each taxa across two samples by the branch length from constructed evolutionary trees [35]. Therefore, the distance between two samples might be larger under gUniFrac if the contributing taxa are evolutionarily divergent. This suggests that samples with 
similar metabolic profiles might be numerically comparable (cluster together under Euclidean distances) but with evolutionarily divergent taxonomic compositions. This supports the hypothesis that phylogenetically distant gut microbes can replace each other while performing similar roles, a hallmark of functional redundancy [33, 36]. Since this was only observed in the targeted data set, we contend that the effect of redundancy is localized to certain metabolites and is less pronounced globally. Functional redundancy is also consistent with previous research in human associated microbiomes [37].

\section{Taxa and metabolites selected to be core to the microbiome-metabolome correlation reveal the importance of amino acid metabolism.}

Taxa and metabolites with non-zero loading coefficients in SCCA analyses were identified as factors driving this overall correlation. The SCCA procedure utilized a $\mathrm{L}_{1}$-penalized matrix decomposition of the cross-product matrix akin to a LASSO regression problem [38], which means that variables were selected based on their importance to the overall covariance between taxa and metabolite abundances.

In the targeted data set, Firmicutes, Actinobacteria and Proteobacteria were the most represented phyla across both targeted and untargeted data sets, consistent with the major phyla present in infant guts compared to adults [25, 27, 39]. At 6 weeks, the Firmicutes selected were evenly split between the Clostridia and Bacilli classes, with Peptostreptococcaceae being the most dominant family among Clostridia. Alternatively, at 12 months, most Firmicutes were only of the Clostridia class, mostly represented by the Ruminococcaceae and Lachnospiraceae families. Both Clostridia and Bacilli classes have been shown to be important in the production of SCFAs [40, 41]. SCFAs (which includes compounds such as butyrate, formate, proprionate and lactate) are important metabolites obtained primarily from colonic microbial fermentation of carbohydrates that escape digestion in the small intestines [42]. Butyrate, for example, is an energy source for colonocytes [43] as well as participating in the maintenance of the gut epithelial barrier through mucin production [44]. Similarly, propionate is part of the gluconeogenesis pathway in liver hepatocyte cells, which is core to lipid and energy metabolism in liver [45]. Most importantly, SCFAs participate in immune programming in early life, where the reduction in SCFA producing bacteria is associated with inflammatory bowel disease [46, 47]. SCFA 
production in early life is linked to the Bifidobacterium and Bacteroides catabolism of human milk oligosaccharides (HMO) [48-50], which explains the selection of the Bifidobacterium genera at 6 weeks where infants are exclusively on a milk-based diet. The selection of SCFA-associated bacteria further demonstrates the functional role that SCFA play in the interface between the microbiome and the host [42]. This is further supported by the fact that two SCFAs in our targeted list (butyrate and propionate) were selected as important variables in SCCA analyses.

Despite the importance of SCFAs, the most selected metabolites are of the amino acids class (7 out of 10 metabolites selected at 6 weeks were amino acids). Although microbes can catabolize amino acids for energy [51], this reaction is not energetically efficient [10]. However, microbes will process amino acids in environments with low carbohydrate availability, such as in the distal colon $[9,52]$, where one of the end point products is SCFAs. This is further supported by the fact that members of the Peptostreptococcaceae family (most represented family of selected members of the Clostridia class at 6 weeks) has been suggested to be core to the metabolism of amino acids to SCFAs [51]. However, looking across both time points in our analysis, negative correlation with amino acids did not correspond to a positive association with SCFAs (Figure 3), suggesting that amino acid depletion in the fecal metabolome is most likely either to conserve resources from reducing amino acid biosynthesis [10], generate energy in a low carbohydrate environment, or reduce toxic byproducts of protein catabolism [53] rather than to produce SCFAs. However, there could be a possibility that SCFA was produced but rapidly absorbed, which could not be captured by cross-sectional fecal metabolomics.

\section{The microbiome is more tightly coupled with the metabolome in early infancy}

Results suggest some level of significant difference in microbiome-metabolome coupling across development. Canonical correlation, while not significantly different, were lower at 12 months than at 6 weeks, suggesting a time-varying effect. When looking at predictability, we observed a higher number of well predicted metabolites at 6 weeks compared to 12 months. Among those selected as well predicted metabolites, the average performance values (both $\mathrm{R}^{2}$ and SCC) where higher. This is also replicated in the global untargeted data set. 
There are various factors that can contribute to the difference in microbiome-metabolome coupling between infants at 6 weeks and 12 months. First, there exists substantive differences in dietary patterns for those included in our analysis. The majority of infants at 6 weeks (62\%) were exclusively breastfed, while that number is markedly less (35\%) at 12 months, at which time infants are also consuming complimentary solid family foods. This transition in diet to solid foods have been shown to induce a change in the gut microbiome composition and diversity due to increased amounts of fiber and protein $[54,55]$, which might favor certain microbes over others. Such changes in diet, particularly the cessation of breastmilk intake, also contributed towards the development of infant gut microbiomes towards a more "adult like" state [27, 54]. We hypothesized that earlier in life when infants are only consuming a limited type of food (predominantly breast milk or formula), the microbiome participates more actively in host-microbiome co-metabolic activity as infants are more reliant on microbes to breakdown complex nutrients [56]. Conversely, at one year of age where the microbiome has matured, this relationship is not as strongly coupled as a larger share of the metabolome comes from hostproduced metabolites.

However, as analyses were conducted within each timepoint independently with little subject overlap, further investigations are required to make more conclusive statements about the potential time-varying effect of microbiome-metabolome coupling. Particularly, aside from differences in diet, factors such as differences in antibiotic exposure [57] and maternal covariates [58] might result in differences between time points. In future studies we hope to examine this factor using samples across multiple time points for the same infants.

\section{Limitations}

This study has various limitations. First, we utilized partial 16S rRNA gene sequencing instead of shotgun whole genome sequencing, which removes our ability to incorporate the functional metagenome into the microbiome/metabolome interface, as well as limiting our taxonomic resolution to the genus level for most of the analysis [59]. We hypothesized this lack of resolution contribute to overall lack of predictability, as well as limiting the interpretability of variables selected by the SCCA process as species and strain level differences can result in completely separate metabolic contributions [60]. 
Second, our cohort includes only infants from the NHBCS, a population-based cohort reflecting mostly rural and White demographics of northern New England in the United States. While this increases confidence in the internal validity of our study, this homogeneity in race and geography limits the generalizability of our results to other populations.

Third, our study is a cross-sectional survey if microbiome-metabolome relationships at two different time points. This means that we cannot capture associations relating to metabolites that are highly produced and consumed. This means that the metabolites selected might not be representative of the intricate relationship between the microbiome and the metabolome. This interpretation is further limited by the lack of annotation for our untargeted metabolite bins, which cannot be compensated by the small number of metabolites selected for the targeted analyses.

Finally, each time point was analyzed independently with only 65 subjects with samples in both time points. As such, this limits the ability to explore the differences in coupling across the first year of life.

\section{Conclusion}

In conclusion, we conducted one of the first large-scale multi-omics analysis of the microbiome-metabolome relationship using samples from a large birth cohort study at 2 time points (6 weeks and 12 months). Although we found global concordance between the microbiome and the metabolome, the inter-omic concordance is weak, where bacterial abundances cannot predict metabolite concentrations. In addition to the involvement of host-produced metabolites as part of the metabolome, we suggest the role of functional redundancy as a possible mechanism behind the lack of coupling identified. Additionally, we were able to identify metabolites and microbes driving the overall correlation. Results pointed to support the importance of SCFA metabolism particularly at 6 weeks, as well as the role of amino acid metabolism, either as a source of SCFA and energy in the absence of carbohydrates, or as a general mechanism for microbes to save energy as they incorporate amino acids around their environment. 
402

403

404

405

406

407

408

409

410

411

412

We conclude that although the metabolome is a functional output of the microbiome, there exists massive challenges in being able to trace specific microbial contributions to host-microbe metabolism due to the complexity of factors such as functional redundancy. As such, we recommend studies to profile both the microbiome and the metabolome, as aspects of microbial metabolic contributions cannot be found solely through one omic data set. This is particularly important in settings where it is important to have a mechanistic understanding of the role of microbes such as developing of microbiome therapies [61].

\section{Methods}

\section{Study population}

Subjects for this study were from the New Hampshire Birth Cohort Study (NHBCS) who provided infant stool samples at 6-weeks and 12-months after birth. As described in previous studies [25, 58], NHBCS is a prospective study of mother-infant dyads in New Hampshire, USA. Participants eligible are pregnant women between the ages of 18 and 45 years old, currently receiving routine prenatal care at one of the study clinics, consuming water out of a private well with no intention to move prior to delivery. The Center for the Protection of Human Subjects at Dartmouth provided institutional review board approval. All methods were carried out in accordance with guidelines. Written informed consent was obtained for participation from all subjects for themselves and their children. Comprehensive sociodemographic, exposure and outcome data such as infant feeding method, delivery mode, maternal smoking status, etc. were collected for all participants through surveys, medical records and telephone interviews conducted during pregnancy, about 6 weeks postpartum, and updated every 4 months up until first year of age and every 6 months thereafter.

\section{Collection of infant stool samples}

Infant stool samples were collected at 6-weeks and 12-months. Stool samples were provided in diapers and stored by subjects in their home freezer $\left(-20^{\circ} \mathrm{C}\right)$ until they were able to return it to the study site. Stool was thawed at $4{ }^{\circ} \mathrm{C}$ so that it could be aliquoted into cryotubes. Stools collected for $16 \mathrm{~S}$ rRNA gene sequencing were aliquoted (range $350-850 \mathrm{mg}$ ) into $3 \mathrm{ml}$ RNAlater and homogenized before storing at $-80^{\circ} \mathrm{C}$. Stools 
426

collected for metabolomic analysis were aliquoted (1-2 grams) into $15 \mathrm{ml}$ centrifuge tubes before storing at -80 ${ }^{\circ} \mathrm{C}$.

\section{Taxonomic profiling using 16S rRNA targeted gene sequencing}

RNAlater stool samples were thawed and DNA was extracted using the Zymo Fecal DNA extraction kit (Cat\# D6010, Zymo Research, Irvine, CA), according to the manufacturer's instructions. For each sample extraction, 400ul RNAlater stool slurry (50-100 mg of stool) was used to isolate DNA. Extractions were performed in batches of multiple samples and included a composite RNAlater stool positive control and a RNAlater negative control. Lysis was performed using 750ul Lysis Buffer in ZR BashingBead ${ }^{\mathrm{TM}}$ Lysis Tubes (0.5 mm beads), mixed and then shaken on a Disruptor Genie for 6 min. Eluted DNA was quantified on a Qubit ${ }^{\text {TM }}$ fluorometer using the Qubit ${ }^{\mathrm{TM}}$ dsDNA BR Assay. Average coefficient of variation of DNA yields (ng/ul) for composite RNAlater stool positive controls was $28 \%$. No DNA was ever detectable in negative control elutions. Concentrations of DNA samples used for 16S rRNA gene sequencing range from $1 \mathrm{ng} / \mathrm{ul}$ to $25 \mathrm{ng} / \mathrm{ul}$.

The V4-V5 hypervariable region of bacterial 16S rRNA gene was sequenced at Marine Biological Laboratory in Woods Hole, MA, using standard approaches [62, 63]. As described previously $[25,58], 16 S$ rDNA V4-V5 amplicons were generated from purified genomic DNA samples using fusion primers. The use of forward primers containing one of eight five-nucleotide barcodes between the Illumina-specific bridge and sequencing primer regions and the 16S-specific region and a single reverse primer containing 1 of 12 Illumina indices enables 96 samples per lane multiplexing. Amplifications were done in triplicate with one negative control for internal quality control at MBL. We used qPCR (Kapa Biosystems) to quantify the amplicon pool, and one Illumina MiSeq 500 cycle paired end run to sequence each pool of 96 libraries. We demultiplex and divided datasets using Illumina MiSeq reporter and a custom Python script. Demultiplexed reads derived from Illumina sequencing were denoised and quality filtered using DADA2 (v. 1.12.1) [64] in R (v. 3.6.1) [65]. Illumina adapter sequences were removed prior using cutadapt (v. 1.18). We then use the RDP classifier implemented 
natively in the DADA2 R package with SILVA database (v. 128) to profile the taxonomy of identified amplicon sequence variants (ASVs).

\section{Functional profiling using untargeted and targeted ${ }^{1} \mathrm{H}$ NMR metabolomics}

${ }^{1} \mathrm{H}$ NMR metabolomics was performed in collaboration with the NIH Eastern Regional Comprehensive Metabolomics Resource Core (RCMRC) at UNC Chapel Hill. De-identified stool aliquots were shipped on dry ice and immediately stored at $-80^{\circ} \mathrm{C}$ for metabolomics analysis. Samples were thawed and $\sim 150 \mathrm{mg}$ of stool samples were transferred to MagNA Lyser tubes after recording the weight. Samples were then homogenized with $50 \%$ acetonitrile in water by using the Omni Bead Disruptor (Omni International, GA, USA). Homogenized samples were centrifuged at $16000 \mathrm{rcf}$ and the supernatant was separated into another tube. An aliquot (1000 uL, $100 \mathrm{mg}$ equivalent of fecal mass) was transferred into an Eppendorf tube and lyophilized overnight. The dried extract was reconstituted in $700 \mathrm{uL}$ of NMR master mix (containing 0.2M phosphate buffer, $0.5 \mathrm{mM}$ DSSd6 (internal standard), and $0.2 \%$ sodium azide (preventing bacterial growth)), vortexed on a multi tube vortexer at speed 5 for $2 \mathrm{~min}$ and centrifuged at $16000 \mathrm{rcf}$ for $5 \mathrm{~min}$. A $600 \mu \mathrm{l}$ aliquot of the supernatant was transferred into pre-labeled 5mm NMR tubes. Additionally, study pooled quality control (QC) samples (created from randomly selected study samples) and batch pooled QC samples were generated from supernatants of study samples and aliquots of supernatants were dried and reconstituted similar to study samples described above and used for QC purposes.

${ }^{1} \mathrm{H}$ NMR spectra of feces extracts were acquired on a Bruker $700 \mathrm{MHz}$ NMR spectrometer using a $5 \mathrm{~mm}$ cryogenically cooled ATMA inverse probe and ambient temperature of $25 \mathrm{C}$. A 1D NOESY presaturation pulse

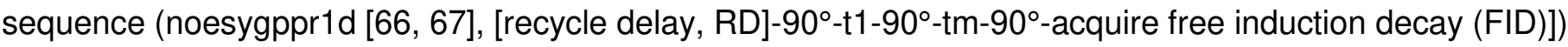
was used for data acquisition. For each sample, 64 transients were collected into 64k data points using a spectral width of $12.02 \mathrm{ppm}, 2 \mathrm{~s}$ relaxation delay, $10 \mathrm{~ms}$ mixing time, and an acquisition time of $3.899 \mathrm{~s} \mathrm{per}$ FID. The water resonance was suppressed using resonance irradiation during the relaxation delay and mixing time. NMR spectra were processed using TopSpin 3.5 software (Bruker-Biospin, Germany). Spectra were zero filled, and Fourier transformed after exponential multiplication with line broadening factor of 0.5 . Quality control 
measures included review of each NMR spectrum for line shape and width, phase and baseline of spectra, and tight clustering of QC samples in Principal Component Analysis [68]. NMR bin data (0.49-9.0 ppm) were generated (untargeted data) excluding water (4.73-4.85 ppm) using intelligent bucket integration of $0.04 \mathrm{ppm}$ bucket width with $50 \%$ looseness using ACD Spectrus Processor (ACD Labs Inc, Toronto, Canada).The integrals of each bin were normalized to the total spectral intensity of each spectrum and transferred to analysis software. This resulted in a collection of spectral bins with bin-specific relative abundances, which will be called the untargeted data. In addition, relative concentration of library-matched metabolites (selected from the literature implicated to be important in host-microbe relationships - Table S1) was determined by using Chenomx NMR Suite 8.4 Professional software [26].This data set will be called the targeted data set.

\section{Software and tools}

All analyses were performed using the R programming language (v. 3.6.3) [65] and associated packages. All data wrangling steps were performed using phyloseq [69], plyr and tidyverse packages [70], as well as the compositions package [71] for log-ratio transformations. All figures were generated using the ggplot2 [72], cowplot [73], viridis [74] and pheatmap [75] packages. Additionally, the tidymodels [76] suite of packages was utilized to assist in all modelling tasks. Specific packages used for modelling will be enumerated below. All scripts as well as intermediary analysis objects are available on github with all dependencies and their versions (https://github.com/qpmnguyen/infant metabolome microbiome).

\section{Data transformation and normalization}

For microbiome data, we retained all ASVs present in at least $10 \%$ of samples [29] and added one pseudocount to all cells [77]. We then subsequently aggregated all ASVs to the genus taxonomic level [28] and converted data to relative proportions using total read counts by sample to account for differential sequencing depth. We further filtered out taxa with mean relative proportion $<0.005 \%$ [78]. To address the compositional problem induced by a sum to one constraint, we apply the centered log ratio transformation (CLR), which is often used to remove such constraints in microbiome data sets [79]. 
498

Given $w$ as a compositional vector with elements $w_{1}, \ldots, w_{p}$ where $\sum_{1}^{p} w_{i}=1$, we can define the centered log ratio transformation $\operatorname{clr}(w)$ as $z$ :

$$
z=\left[\log \left(\frac{w^{1}}{g(w)}\right), \ldots, \log \left(\frac{w^{p}}{g(w)}\right)\right]
$$

where $g(w)=\left[\prod_{i=1}^{p} w^{j}\right]^{\frac{1}{p}}$ is the geometric mean of the composition. Consequently, $z$ exists in a $p-1$ dimensional Euclidean space and is constrained to sum to 0 . The CLR transformation is favored compared to other statistically equivalent log-ratio transformations due to its scale invariant property and ease of interpretation [80].

For metabolomic data sets, we employed different transformations to approximate homoscedasticity depending on the data type (targeted vs untargeted). For targeted metabolites, we performed a $\log (x+1)$ transformation while for untargeted metabolites we utilized the arcsine square root transformation which has been previously used for transforming composition metabolomics data sets [29].

\section{Distance matrix analyses}

Principal coordinates analysis (PCoA) was performed using the pcoa function from the ape package in $\mathrm{R}$ [81] with sample distance matrices. The PCoA procedure seeks to represent high dimensional multivariate data sets in lower dimensions through eigen decomposition of the doubly centered distance matrix. PCoA allows the usage of non-Euclidean distances between samples such as ecological indices, which makes it a preferable method for sample ordination compared to principal component analysis (PCA).

We constructed Euclidean distance matrices for both metabolic and taxonomic profiles post data transformation described in the previous section. Additionally, gUniFrac distances (alpha $=0.5)$ [62] were considered for taxonomic data using the implementation provided in the package MiSPU [63]. gUniFrac requires a phylogenetic tree, of which an approximate maximum likelihood phylogenetic tree was constructed with representative ASV sequences using FastTree (v 2.1) [64]. Multiple sequence alignment was performed using the AlignSeqs function from the DECIPHER package in $\mathrm{R}[65]$ and trees were midpoint rooted using 
phytools [66]. Since multiple sequence alignment is not conserved under filtering and aggregation of ASVs, gUniFrac distance calculations were performed with pre-filtered ASV-level abundances normalized to relative abundances.

The first two axes of constructed ordinations were then compared using a symmetric Procrustes procedure implemented in the protest function in the vegan package [67]. Procrustes superimposes two ordinations by translating and rotating the coordinates, which preserves the general structure of the data. This method performs a superimposition fit between two data sets minimizing the sum-of-squared differences $\left(\mathrm{m}^{2}\right)$, which describes the degree of concordance between the two configurations normalized to unit variance. Significance is obtained by testing against the permuted null using a permutation test. This method was shown to have more power while also limiting type I error compared to the traditional Mantel test in ecological analysis [82]. Significance was determined using a permutation test on the sum of squared differences with 999 permutations [68].

\section{Sparse canonical correlation and Spearman correlation analyses}

Sparse canonical correlation analysis ( $\mathrm{SCCA}$ ) was performed to identify strongly associated metabolitemicrobe groups. SCCA seeks to find linear combinations of variables from each dataset that maximizes the correlation with each other while simultaneously thresholding variable specific weights to induce sparsity and performing variable selection. The correlation coefficient in the first canonical variate quantifies the overall degree of multivariate associations. As such, sCCA is a popular method in integrating multi-omics datasets with the ability to select more biologically relevant sets of features compared to traditional ecological methods such as co-inertia analysis [83]. In this study, we use the sCCA implementation in the package PMA in R [38] which uses a novel penalized matrix decomposition procedure to achieve sparsity [84]. We tune hyperparameters using a permutation approach in the CCA.permute function (nperms $=50$ ) prior to fitting the final model. We obtain the correlation coefficients as a measure of overall correlation between the two data sets and calculated a bootstrapped $95 \%$ confidence interval $(n b o o t=5000)$ as well as performing a permutation test $(\mathrm{nperm}=1000)$ at the 0.05 significance level. In order to keep the structure of the data set 
across different permutations, we use the function randomizeMatrix from the package picante in $\mathrm{R}$ [85] using the richness null model, which randomizes community abundances within samples to maintain sample species richness.

Pairwise Spearman correlations were also performed using the cor function in R. Hypothesis testing was done using cor.test, with multiple hypothesis testing correction using the Benjamini-Hochberg procedure using p.adjust. An FDR value of 0.05 is used as cutoff for significance pairwise correlations. Visualization was done using pheatmap package in $\mathrm{R}$.

\section{Predictive modelling and evaluation}

We choose candidate models based on previous research utilizing supervised learning with microbiome associated prediction tasks [28, 29, 31]. Specifically, we chose random forest (RF) [86], support vector machine with radial basis function kernel (SVM-RBF) [87], elastic net (EN) [88] and sparse partial least squares (SPLS) [89], which have all been shown to perform with high-dimensional predictors. These models also support linear and non-linear associations between the microbiome and the outcome of interest. Model fitting, parameter tuning, and evaluation were done using caret package in $\mathrm{R}[90]$. Parallel processing was performed using the doParallel [91] and parallel packages.

We evaluate prediction performance by performing 100 repeats of 10 -fold nested cross validation, whereby within each training fold is a separate 5-fold cross-validation procedure done to perform hyperparameter selection when appropriate with parameter grids modelled after Pasolli et al. [31]. For RF, we set the number of trees to be 500 , and the number of features used in each decision tree to be the square root of the number of the original features. For SVM-RBF, we tuned across a grid for the regularization parameter $C$ (values $\left.2^{-5}, 2^{-3}, \ldots, 2^{15}\right)$ and the kernel width parameter $\mathrm{Y}\left(\right.$ values $\left.2^{-15}, 2^{-13}, \ldots, 2^{3}\right)$. For EN, we tuned over a grid of the regularization parameter $\lambda$ and the $L_{1}$ to $L_{2}$ penalty ratio $\alpha$, where for each $\alpha$ value (spaced by 0.1 ) between 0 (equivalent to a LASSO model) and 1 (equivalent to a ridge regression model), we evaluate 100 lambda values chosen by the glmnet procedure. For SPLS, we kept the concavity parameter $\mathrm{k}$ constant at 0.5 while tuning the number of components $K$ (values $1,2, \ldots, 10)$ and the thresholding parameter $\eta$ (values $0.1,0.2, \ldots, 0.9$ ). 
We utilize standard regression evaluation metrics include predictive $R$-squared $\left(R^{2}\right)$ and Spearman correlation coefficient (SCC) which are defined as:

$$
\begin{gathered}
R^{2}=1-\frac{\sum_{1}^{n}\left(y_{i}-\hat{y}_{i}\right)^{2}}{\sum_{i}^{n}\left(y_{i}-\bar{y}\right)^{2}}(y \text { and } \hat{y} \text { are true and predicted values }) \\
S C C=\frac{\operatorname{cov}\left(y_{(i)}, \hat{y}_{(i)}\right)}{\sigma_{y_{(i)}} \sigma_{\hat{y}_{(i)}}}\left(y_{(i)} \text { and } \hat{y}_{(i)}\right. \text { are ranked true values and predicted values) }
\end{gathered}
$$

These statistics were chosen due to their ability to capture two different aspects of the regression task.

Predictive $R^{2}$ captures the predicted residual sum of squares (PRESS) normalized by the total sum of squares of the outcome, thereby measuring predictive performance while also putting it into context of a naive, intercept only model. On the other hand, SCC quantifies the monotonic association between true and predicted values, providing perspective as to whether or not the predicted values can capture the overall trend of the outcome. Prior to evaluation, all metabolites were back transformed to their original scale. In order to perform comparisons between models across time points and metabolites as well as ascertaining the uncertainty of each evaluation metric, a Bayesian approach as presented in [92]. Specifically, a generalized Bayesian hierarchical linear model (with identity link and gaussian standard error) in the following form was fitted for each metabolite:

$$
\text { EvaluationStatistic } \sim \text { Model }+(1 \mid \text { repeat })+(1 \mid \text { repeat }: \text { fold })
$$

This model assumes that the distribution of the evaluation statistic as a linear function of model assignment, with random intercepts varying among repeats and for folds within each repeat. Models were fitted using implementation in the R package tidyposterior [93] using default weakly informative priors as described in the rstanarm package [94]. Using this model, a predictive posterior mean and $95 \%$ credible interval can be generated. The posterior mean is then used to rank the best performing model for each metabolite according to the evaluation metric of interest. Ranks are then aggregated using the Borda method [95] to generate Borda scores. In detail, for each metabolite, 4 points are added to the top ranked model, 3 points to the second ranked model and so on. The model with the highest total points for each metric is the most performant model aggregated across all prediction tasks. 


\section{Simulation design}

595 Simulations were performed to examine the behavior of models under known association/null settings in order 596 to validate findings. For the first simulation scenario, a linear association between genus-level taxonomic abundance and log transformed metabolite concentrations were simulated. The predictor matrix were bootstrapped resamples of the community matrix post data processing. $\beta$ coefficient values were sampled from the standard normal distribution $N(0,1)$ values for each genus would have a probability $p(0.05,0.1,0.5,0.95)$ of being 0 which determines the sparsity of the coefficients (or the level of model saturation). We generate metabolite outcome values $Y$ following the model

$$
Y=\beta_{0}+X \beta+\epsilon
$$

where $X$ is the $n \times p$ simulated taxonomic predictor matrix, $\beta$ is the $p \times 1$ previously defined coefficient vector, $\epsilon \sim N\left(\mu=0, \sigma=\sigma_{\epsilon}\right)$ is the standard normal noise vector. Similar to Xiao et al. 2018 [96] and Shi et al. 2016

[97], we set all $\beta_{0}=\frac{6}{\sqrt{10}}$ and $\sigma_{\epsilon}=\frac{\sigma\left(\beta_{0}+X \beta\right)}{S N R}$ where signal-to-noise ratio (SNR) are set at $0.5,0.7,3,5$ to simulate both situations where noise is higher than signal and vice versa. For each simulation setting, 100 data sets were generated.

For the second simulation scenario, null models were assessed through a permutation procedure using the picante package in $\mathrm{R}$ as described earlier. A total of 500 permutations was performed for each model.

To evaluate the predictive capacity of models for each simulation scenario, each data set was split into a train and test set ( $80 \%$ train; $20 \%$ test). Within each training set, a 10 -fold cross validation procedure was employed to tune any hyperparameters. Similar evaluation metrics were assessed as described in the model fitting section.

\section{List of abbreviations}

NHBCS: New Hampshire Birth Cohort Study

NMR: Nuclear Magnetic Resonance 
616 PCoA: Principal Coordinates Analysis

617 gUniFrac: Generalized Unique Fraction

618 ASV: Amplicon Sequence Variant

619 FDR: False Discovery Rate

620 sCCA/CCA: Sparse Canonical Correlation Analysis

621 SCFA: Short Chain Fatty Acids

622 RF: Random Forest

623 EN: Elastic Net

624 SVM-RBF: Support Vector Machines with Radial Basis kernel Function

625 SPLS: Sparse Partial Least Squares

626 CLR: Centered Log Ratio transformation

\section{Additional Files}

628 Additional File 1: Contains supplementary notes (Notes $1-3$ ), supplementary figures (Figures S1-S8) and

629 supplementary table 1. (DOCX 2429KB)

630 Additional File 2: Pairs of microbes and targeted metabolites with significant spearman correlations at q-value

$631<0.05$ with their correlation values for each time point. (XLS 22KB)

Additional File 3: List of targeted metabolites with predictive $R^{2}>0$ or spearman correlation $>0.3$ for each model across all time points. (XLSX 17KB)

\section{Declarations}

\section{Ethics approval and consent to participate}

636 The Center for the Protection of Human Subjects at Dartmouth provided institutional review board approval. All 637 methods were carried out in accordance with the guidelines. Written informed consent was obtained for 638 participation from all subjects for themselves and their children. 


\section{Availability of data and material}

The 16S rRNA gene sequencing datasets used in this study are stored in the National Center for Biotechnology Information (NCBI) Sequence Read Archive: http://www.ncbi.nlm.nih.gov/sra under accession number PRJNA296814. The raw and processed metabolomics data and associated de-identified metadata are available at the NIH Common Fund Metabolomics Data Repository (www.metabolomicsworkbench.org, Study ID\#: XXXX - in progress). All intermediary analysis objects and scripts are available on Github.

\section{Competing interests}

The authors declare that they have no competing financial interests

\section{Funding}

This research is supported by funding from the National Institutes of Health (grants NLM R01LM012723, NIGMS P20GM104416, NCI P30CA023108, NCI R21CA253408, NLM K01LM012426, NIH UG3 OD023275, NIEHS P01ES022832 and EPA RD-83544201). None of the funding bodies had a role in the design of the study or the collection, analysis, and interpretation of data and in writing of the manuscript.

\section{Authors' contributions}

QPN, AGH and HRF designed the research. QPN performed the statistical analysis and wrote the paper. QPN, AGH, HRF, MRK, and JCM interpreted the results. ED performed bioinformatic processing and maintained the data. TJP performed laboratory extractions of samples. HGM provided technical and methodological support for sequencing data. SM, SJS and WWP provided technical and methodological support for metabolomic. AGH and HRF jointly supervised this research. AGH has primary responsibility for all the content. All authors have reviewed the manuscript, offered critical feedback, and approved the final version.

\section{Acknowledgements}

We thank the participants of the New Hampshire Birth Cohort Study and to the study staff, without whom this research would not be possible. We would also like to thank all members of the Microbiome research group at Dartmouth College for all advice and additional assistance in preparing this manuscript.

\section{Authors' Information}

\section{Department of Epidemiology, Geisel School of Medicine at Dartmouth, Hanover, NH, USA}


66

Quang P. Nguyen, Erika Dade, Tom J. Palys, Juliette C. Madan, Margaret R. Karagas \& Anne G. Hoen Division of Neonatology, Department of Pediatrics, Children's Hospital at Dartmouth, Hanover, NH, USA

Juliette C. Madan

Children's Environmental Health \& Disease Prevention Research Center at Dartmouth, Lebanon, NH, USA

Juliette C. Madan, Margaret R. Karagas \& Anne G. Hoen

Department of Biomedical Data Science, Geisel School of Medicine at Dartmouth, Hanover, NH, USA Anne G. Hoen \& H. Robert Frost

Josephine Bay Paul Center, Marine Biological Laboratory, Woods Hole, MA, USA

Hilary G. Morrison

Department of Nutrition, Nutrition Research Institute, University of North Carolina at Chapel Hill, Chapel Hill, NC, USA

Wimal W. Pathmasiri, Susan McRitche \& Susan J. Sumner

\section{References}

1. Shreiner AB, Kao JY, Young VB. The gut microbiome in health and in disease. Curr Opin Gastroenterol. 2015;31:69-75.

2. Palmer C, Bik EM, DiGiulio DB, Relman DA, Brown PO. Development of the Human Infant Intestinal Microbiota. PLOS Biol. 2007;5:e177.

3. Koenig JE, Spor A, Scalfone N, Fricker AD, Stombaugh J, Knight R, et al. Succession of microbial consortia in the developing infant gut microbiome. Proc Natl Acad Sci. 2011;108 Supplement 1:4578-85. 
687

688

689

690

691

692

693

694

695

696

697

698

699

700

701

702

703

704

705

706

70

4. Stanislawski MA, Dabelea D, Wagner BD, Iszatt N, Dahl C, Sontag MK, et al. Gut Microbiota in the First 2 Years of Life and the Association with Body Mass Index at Age 12 in a Norwegian Birth Cohort. mBio. 2018;9. doi:10.1128/mBio.01751-18.

5. Kostic AD, Gevers D, Siljander H, Vatanen T, Hyötyläinen T, Hämäläinen A-M, et al. The Dynamics of the Human Infant Gut Microbiome in Development and in Progression toward Type 1 Diabetes. Cell Host Microbe. 2015;17:260-73.

6. Arrieta M-C, Stiemsma LT, Amenyogbe N, Brown EM, Finlay B. The Intestinal Microbiome in Early Life: Health and Disease. Front Immunol. 2014;5. doi:10.3389/fimmu.2014.00427.

7. Heintz-Buschart A, Wilmes P. Human Gut Microbiome: Function Matters. Trends Microbiol. 2018;26:563-74.

8. Turnbaugh PJ, Gordon JI. An invitation to the marriage of metagenomics and metabolomics. Cell. $2008 ; 134: 708-13$.

9. Ewald DR, Sumner SC. Human Microbiota, Blood Group Antigens, and Disease. Wiley Interdiscip Rev Syst Biol Med. 2018;10:e1413.

10. Oliphant K, Allen-Vercoe E. Macronutrient metabolism by the human gut microbiome: major fermentation by-products and their impact on host health. Microbiome. 2019;7:91.

11. Heinken A, Thiele I. Systems biology of host-microbe metabolomics. Wiley Interdiscip Rev Syst Biol Med. 2015;7:195-219.

12. Li M, Wang B, Zhang M, Rantalainen M, Wang S, Zhou H, et al. Symbiotic gut microbes modulate human metabolic phenotypes. Proc Natl Acad Sci. 2008;105:2117-22.

13. Nicholson JK, Holmes E, Kinross J, Burcelin R, Gibson G, Jia W, et al. Host-Gut Microbiota Metabolic Interactions. Science. 2012;336:1262-7. 
14. Pedersen HK, Gudmundsdottir V, Nielsen HB, Hyotylainen T, Nielsen T, Jensen BAH, et al. Human gut microbes impact host serum metabolome and insulin sensitivity. Nature. 2016;535:376-81.

15. Neis EPJG, Dejong CHC, Rensen SS. The Role of Microbial Amino Acid Metabolism in Host Metabolism. Nutrients. 2015;7:2930-46.

16. Zierer J, Jackson MA, Kastenmüller G, Mangino M, Long T, Telenti A, et al. The fecal metabolome as a functional readout of the gut microbiome. Nat Genet. 2018;50:790-5.

17. Lloyd-Price J, Arze C, Ananthakrishnan AN, Schirmer M, Avila-Pacheco J, Poon TW, et al. Multi-omics of the gut microbial ecosystem in inflammatory bowel diseases. Nature. 2019;569:655-62.

18. Wandro S, Osborne S, Enriquez C, Bixby C, Arrieta A, Whiteson K. The microbiome and metabolome of pre-term infant stool is personalized, and not driven by health outcomes including necrotizing enterocolitis and late-onset sepsis. 2018.

19. Ayeni FA, Biagi E, Rampelli S, Fiori J, Soverini M, Audu HJ, et al. Infant and Adult Gut Microbiome and Metabolome in Rural Bassa and Urban Settlers from Nigeria. Cell Rep. 2018;23:3056-67.

20. Stewart CJ, Embleton ND, Marrs ECL, Smith DP, Fofanova T, Nelson A, et al. Longitudinal development of the gut microbiome and metabolome in preterm neonates with late onset sepsis and healthy controls. Microbiome. 2017;5. doi:10.1186/s40168-017-0295-1.

21. Younge NE, Newgard CB, Cotten CM, Goldberg RN, Muehlbauer MJ, Bain JR, et al. Disrupted Maturation of the Microbiota and Metabolome among Extremely Preterm Infants with Postnatal Growth Failure. Sci Rep. 2019;9:1-12.

22. Brink L, Chintapalli S, Mercer K, Piccolo B, Adams S, Bowlin A, et al. Early Postnatal Diet Differentially Affects the Fecal Microbiome and Metabolome (FS04-02-19). Curr Dev Nutr. 2019;3 Supplement_1. doi:10.1093/cdn/nzz048.FS04-02-19. 
730

731

732

733

734

735

736

737

738

739

740

741

742

743

744

745

746

747

748

749

750

23. Kisuse J, La-ongkham O, Nakphaichit M, Therdtatha P, Momoda R, Tanaka M, et al. Urban Diets Linked to Gut Microbiome and Metabolome Alterations in Children: A Comparative Cross-Sectional Study in Thailand. Front Microbiol. 2018;9. doi:10.3389/fmicb.2018.01345.

24. Hill CJ, Lynch DB, Murphy K, Ulaszewska M, Jeffery IB, O'Shea CA, et al. Evolution of gut microbiota composition from birth to 24 weeks in the INFANTMET Cohort. Microbiome. 2017;5:4.

25. Madan JC, Hoen AG, Lundgren SN, Farzan SF, Cottingham KL, Morrison HG, et al. Effects of Cesarean delivery and formula supplementation on the intestinal microbiome of six-week old infants. JAMA Pediatr. 2016;170:212-9.

26. Weljie AM, Newton J, Mercier P, Carlson E, Slupsky CM. Targeted Profiling: Quantitative Analysis of ${ }^{1} \mathrm{H}$ NMR Metabolomics Data. Anal Chem. 2006;78:4430-42.

27. Bäckhed F, Roswall J, Peng Y, Feng Q, Jia H, Kovatcheva-Datchary P, et al. Dynamics and Stabilization of the Human Gut Microbiome during the First Year of Life. Cell Host Microbe. 2015;17:690-703.

28. Zhou Y-H, Gallins P. A Review and Tutorial of Machine Learning Methods for Microbiome Host Trait Prediction. Front Genet. 2019;10. doi:10.3389/fgene.2019.00579.

29. Mallick H, Franzosa EA, Mclver LJ, Banerjee S, Sirota-Madi A, Kostic AD, et al. Predictive metabolomic profiling of microbial communities using amplicon or metagenomic sequences. Nat Commun. 2019;10:1-11.

30. McHardy IH, Goudarzi M, Tong M, Ruegger PM, Schwager E, Weger JR, et al. Integrative analysis of the microbiome and metabolome of the human intestinal mucosal surface reveals exquisite inter-relationships. Microbiome. 2013;1:17.

31. Pasolli E, Truong DT, Malik F, Waldron L, Segata N. Machine Learning Meta-analysis of Large Metagenomic Datasets: Tools and Biological Insights. PLOS Comput Biol. 2016;12:e1004977. 
32. Shaffer M, Thurimella K, Quinn K, Doenges K, Zhang X, Bokatzian S, et al. AMON: annotation of metabolite origins via networks to integrate microbiome and metabolome data. BMC Bioinformatics. 2019;20:614.

33. Louca S, Polz MF, Mazel F, Albright MBN, Huber JA, O'Connor MI, et al. Function and functional redundancy in microbial systems. Nat Ecol Evol. 2018;:1.

34. Allison SD, Martiny JBH. Resistance, resilience, and redundancy in microbial communities. Proc Natl Acad Sci. 2008;105 Supplement 1:11512-9.

35. Chen J, Bittinger K, Charlson ES, Hoffmann C, Lewis J, Wu GD, et al. Associating microbiome composition with environmental covariates using generalized UniFrac distances. Bioinformatics. 2012;28:2106-13.

36. Vieira-Silva S, Falony G, Darzi Y, Lima-Mendez G, Yunta RG, Okuda S, et al. Species-function relationships shape ecological properties of the human gut microbiome. Nat Microbiol. 2016;1:16088.

37. Lozupone CA, Stombaugh JI, Gordon JI, Jansson JK, Knight R. Diversity, stability and resilience of the human gut microbiota. Nature. 2012;489:220-30.

38. Witten D, Tibshirani R, Gross S, Narasimhan B. PMA: penalized multivariate analysis. 2019. https://CRAN.R-project.org/package=PMA.

39. Stewart CJ, Ajami NJ, O'Brien JL, Hutchinson DS, Smith DP, Wong MC, et al. Temporal development of the gut microbiome in early childhood from the TEDDY study. Nature. 2018;562:583-8.

40. Lopetuso LR, Scaldaferri F, Petito V, Gasbarrini A. Commensal Clostridia: leading players in the maintenance of gut homeostasis. Gut Pathog. 2013;5:23.

41. Ilinskaya ON, Ulyanova VV, Yarullina DR, Gataullin IG. Secretome of Intestinal Bacilli: A Natural Guard against Pathologies. Front Microbiol. 2017;8. doi:10.3389/fmicb.2017.01666. 
42. Morrison DJ, Preston T. Formation of short chain fatty acids by the gut microbiota and their impact on human metabolism. Gut Microbes. 2016;7:189-200.

43. LeBlanc JG, Chain F, Martín R, Bermúdez-Humarán LG, Courau S, Langella P. Beneficial effects on host energy metabolism of short-chain fatty acids and vitamins produced by commensal and probiotic bacteria. Microb Cell Factories. 2017;16. doi:10.1186/s12934-017-0691-z.

44. Peng L, Li Z-R, Green RS, Holzman IR, Lin J. Butyrate Enhances the Intestinal Barrier by Facilitating Tight Junction Assembly via Activation of AMP-Activated Protein Kinase in Caco-2 Cell Monolayers. J Nutr. 2009;139:1619-25.

45. den Besten G, Lange K, Havinga R, van Dijk TH, Gerding A, van Eunen K, et al. Gut-derived short-chain fatty acids are vividly assimilated into host carbohydrates and lipids. Am J Physiol-Gastrointest Liver Physiol. 2013;305:G900-10.

46. Corrêa-Oliveira R, Fachi JL, Vieira A, Sato FT, Vinolo MAR. Regulation of immune cell function by shortchain fatty acids. Clin Transl Immunol. 2016;5:e73.

47. Schulthess J, Pandey S, Capitani M, Rue-Albrecht KC, Arnold I, Franchini F, et al. The Short Chain Fatty Acid Butyrate Imprints an Antimicrobial Program in Macrophages. Immunity. 2019;50:432-445.e7.

48. James K, Bottacini F, Contreras JIS, Vigoureux M, Egan M, Motherway MO, et al. Metabolism of the predominant human milk oligosaccharide fucosyllactose by an infant gut commensal. Sci Rep. 2019;9:1-20.

49. Lawson MAE, O’Neill IJ, Kujawska M, Gowrinadh Javvadi S, Wijeyesekera A, Flegg Z, et al. Breast milkderived human milk oligosaccharides promote Bifidobacterium interactions within a single ecosystem. ISME J. 2020;14:635-48.

50. Marcobal A, Barboza M, Sonnenburg ED, Pudlo N, Martens EC, Desai P, et al. Bacteroides in the Infant Gut Consume Milk Oligosaccharides via Mucus-Utilization Pathways. Cell Host Microbe. 2011;10:507-14. 
794

795

796

797

798

799

800

801

802

803

804

805

806

807

808

809

810

81

812

813

51. Dai Z-L. Amino acid metabolism in intestinal bacteria: links between gut ecology and host health. Front Biosci. 2011;16:1768.

52. Macfarlane GT, Macfarlane S. Bacteria, Colonic Fermentation, and Gastrointestinal Health. J AOAC Int. 2012;95:50-60.

53. Fan P, Li L, Rezaei A, Eslamfam S, Che D, Ma X. Metabolites of Dietary Protein and Peptides by Intestinal Microbes and their Impacts on Gut. Curr Protein Pept Sci. 2015;16:646-54.

54. Moore RE, Townsend SD. Temporal development of the infant gut microbiome. Open Biol. 2019;9:190128.

55. Laursen MF, Bahl MI, Michaelsen KF, Licht TR. First Foods and Gut Microbes. Front Microbiol. 2017;8. doi:10.3389/fmicb.2017.00356.

56. Milani C, Duranti S, Bottacini F, Casey E, Turroni F, Mahony J, et al. The First Microbial Colonizers of the Human Gut: Composition, Activities, and Health Implications of the Infant Gut Microbiota. Microbiol Mol Biol Rev. 2017;81:e00036-17.

57. Coker MO, Hoen AG, Dade E, Lundgren S, Li Z, Wong AD, et al. Specific class of intrapartum antibiotics relates to maturation of the infant gut microbiota: a prospective cohort study. BJOG Int J Obstet Gynaecol. 2020;127:217-27.

58. Lundgren SN, Madan JC, Emond JA, Morrison HG, Christensen BC, Karagas MR, et al. Maternal diet during pregnancy is related with the infant stool microbiome in a delivery mode-dependent manner. Microbiome. 2018;6:109.

59. Zhang C, Zhao L. Strain-level dissection of the contribution of the gut microbiome to human metabolic disease. Genome Med. 2016;8. doi:10.1186/s13073-016-0304-1. 
60. Lloyd-Price J, Mahurkar A, Rahnavard G, Crabtree J, Orvis J, Hall AB, et al. Strains, functions and dynamics in the expanded Human Microbiome Project. Nature. 2017. doi:10.1038/nature23889.

61. Lemon KP, Armitage GC, Relman DA, Fischbach MA. Microbiota-Targeted Therapies: An Ecological Perspective. Sci Transl Med. 2012;4:137rv5.

62. Newton RJ, McLellan SL, Dila DK, Vineis JH, Morrison HG, Eren AM, et al. Sewage Reflects the Microbiomes of Human Populations. mBio. 2015;6. doi:10.1128/mBio.02574-14.

63. Huse SM, Young VB, Morrison HG, Antonopoulos DA, Kwon J, Dalal S, et al. Comparison of brush and biopsy sampling methods of the ileal pouch for assessment of mucosa-associated microbiota of human subjects. Microbiome. 2014;2:5.

64. Callahan BJ, McMurdie PJ, Rosen MJ, Han AW, Johnson AJA, Holmes SP. DADA2: High-resolution sample inference from Illumina amplicon data. Nat Methods. 2016;13:581-3.

65. R Core Team. R: A language and environment for statistical computing. Vienna, Austria; 2019. https://www.R-project.org/.

66. Beckonert O, Keun HC, Ebbels TMD, Bundy J, Holmes E, Lindon JC, et al. Metabolic profiling, metabolomic and metabonomic procedures for NMR spectroscopy of urine, plasma, serum and tissue extracts. Nat Protoc. 2007;2:2692-703.

67. Dona AC, Jiménez B, Schäfer H, Humpfer E, Spraul M, Lewis MR, et al. Precision high-throughput proton NMR spectroscopy of human urine, serum, and plasma for large-scale metabolic phenotyping. Anal Chem. 2014;86:9887-94.

68. Broadhurst D, Goodacre R, Reinke SN, Kuligowski J, Wilson ID, Lewis MR, et al. Guidelines and considerations for the use of system suitability and quality control samples in mass spectrometry assays applied in untargeted clinical metabolomic studies. Metabolomics Off J Metabolomic Soc. 2018;14:72. 
836

837

838

839

840

841

842

843 844 project.org/package $=$ cowplot.

845

846

847

848

849

850

851

852

853

854

855 https://ggplot2.tidyverse.org. project.org/package=tidymodels .

69. McMurdie PJ, Holmes S. phyloseq: An R package for reproducible interactive analysis and graphics of microbiome census data. PLoS ONE. 2013;8:e61217.

70. Wickham H. The split-apply-combine strategy for data analysis. J Stat Softw. 2011;40:1-29.

71. van den Boogaart KG, Tolosana-Delgado R, Bren M. compositions: Compositional data analysis. 2019. https://CRAN.R-project.org/package=compositions.

72. Wickham H. ggplot2: Elegant graphics for data analysis. Springer-Verlag New York; 2016.

73. Wilke CO. cowplot: Streamlined plot theme and plot annotations for "ggplot2." 2019. https://CRAN.R-

74. Garnier S. viridis: Default color maps from “matplotlib.” 2018. https://CRAN.R-project.org/package=viridis.

75. Kolde R. pheatmap: Pretty heatmaps. 2019. https://CRAN.R-project.org/package=pheatmap.

76. Kuhn M, Wickham H. tidymodels: Easily install and load the "tidymodels" packages. 2020. https://CRAN.R-

77. Kurtz ZD, Müller CL, Miraldi ER, Littman DR, Blaser MJ, Bonneau RA. Sparse and Compositionally Robust Inference of Microbial Ecological Networks. PLOS Comput Biol. 2015;11:e1004226.

78. Bokulich NA, Subramanian S, Faith JJ, Gevers D, Gordon JI, Knight R, et al. Quality-filtering vastly improves diversity estimates from Illumina amplicon sequencing. Nat Methods. 2013;10:57.

79. Aitchison J. The Statistical Analysis of Compositional Data. J R Stat Soc Ser B Methodol. 1982;44:139-77.

80. Gloor GB, Macklaim JM, Pawlowsky-Glahn V, Egozcue JJ. Microbiome Datasets Are Compositional: And This Is Not Optional. Front Microbiol. 2017;8. doi:10.3389/fmicb.2017.02224. 
856

858

859

860

861

862

863

864

865

866

867

868

869

870

871

872

873

874

875

81. Paradis E, Schliep K. ape 5.0: an environment for modern phylogenetics and evolutionary analyses in R. Bioinformatics. 2018;35:526-8.

82. Peres-Neto PR, Jackson DA. How well do multivariate data sets match? The advantages of a Procrustean superimposition approach over the Mantel test. Oecologia. 2001;129:169-78.

83. Cao D-S, Liu S, Zeng W-B, Liang Y-Z. Sparse canonical correlation analysis applied to -omics studies for integrative analysis and biomarker discovery. J Chemom. 29:371-8.

84. Witten DM, Tibshirani R, Hastie T. A penalized matrix decomposition, with applications to sparse principal components and canonical correlation analysis. Biostat Oxf Engl. 2009;10:515-34.

85. Kembel SW, Cowan PD, Helmus MR, Cornwell WK, Morlon H, Ackerly DD, et al. Picante: R tools for integrating phylogenies and ecology. Bioinformatics. 2010;26:1463-4.

86. Breiman L. Random Forests. Mach Learn. 2001;45:5-32.

87. Boser BE, Guyon IM, Vapnik VN. A Training Algorithm for Optimal Margin Classifiers. In: Proceedings of the Fifth Annual Workshop on Computational Learning Theory. New York, NY, USA: ACM; 1992. p. $144-152$. doi:10.1145/130385.130401.

88. Zou H, Hastie T. Regularization and Variable Selection via the Elastic Net. J R Stat Soc Ser B Stat Methodol. 2005;67:301-20.

89. Chun H, KeleÅ S. Sparse partial least squares regression for simultaneous dimension reduction and variable selection. J R Stat Soc Ser B Stat Methodol. 2010;72:3-25.

90. Wing MKuhnC from J, Weston S, Williams A, Keefer C, Engelhardt A, Cooper T, et al. Caret: classification and regression training. 2019. https://CRAN.R-project.org/package=caret. 
91. Corporation M, Weston S. doParallel: Foreach parallel adaptor for the "parallel" package. 2019.

877 https://CRAN.R-project.org/package=doParallel.

92. Benavoli A, Corani G, Dem`sar J, Zaffalon M. Time for a Change: a Tutorial for Comparing Multiple Classifiers Through Bayesian Analysis. :36.

93. Kuhn M. tidyposterior: Bayesian analysis to compare models using resampling statistics. 2018. https://CRAN.R-project.org/package=tidyposterior.

94. Brilleman S, Crowther M, Moreno-Betancur M, Buros Novik J, Wolfe R. Joint longitudinal and time-to-event models via Stan. https://github.com/stan-dev/stancon_talks/.

95. Lin S. Rank aggregation methods. Wiley Interdiscip Rev Comput Stat. 2010;2:555-70.

96. Xiao J, Chen L, Yu Y, Zhang X, Chen J. A Phylogeny-Regularized Sparse Regression Model for Predictive Modeling of Microbial Community Data. Front Microbiol. 2018;9. doi:10.3389/fmicb.2018.03112.

97. Shi P, Zhang A, Li H. Regression analysis for microbiome compositional data. Ann Appl Stat. 2016;10:1019-40.

88

\section{Figure and Table Title and Legend}

Figure 1. Overview of the analysis. Panel A describes the subject selection workflow and panel B describes the analytic pipeline.

Table 1. Selected characteristics of subjects contributing samples at 6 weeks $(n=158)$ and at 12 months of age $(n=282)$.

Figure 2: Inter-omics Procrustes biplots comparing PCoA ordinations of targeted metabolite profiles and taxonomic relative abundances for 6 weeks (left panels) $(n=158)$ and 12 months (right panels) $(n=262)$. Top panels present analyses based on ordinations from Euclidean distances of genus level abundances after centered log ratio transformation and Euclidean distances of log-transformed metabolite profiles. Bottom panel 
presents analyses based on gUniFrac distance of amplicon sequence variant (ASV) relative abundances and Euclidean distances of log-transformed metabolite profiles. There were significant associations between the microbiome and the metabolome (both targeted and untargeted) when utilizing Euclidean distances, however this association goes away when the gUniFrac distance was employed for the targeted metabolites only.

Figure 3: Pairwise Spearman correlation of concentration-fitted metabolites and genus-level taxonomic abundances for 6-weeks (panel $A, N=168$ ) and 12-months (panel $B, N=282$ ) infants. Left panel displays the overall correlation pattern, where non-significant correlations are not colored (false discovery rate (FDR) controlled q-value $<0.05)$. Right panel displays the same heatmap restricted to taxa and metabolites selected by the sparse CCA procedure. Additionally, correlation coefficient of the first SCCA variate pair, bootstrapped $95 \%$ confidence interval and permutation $p$-value are also reported. Significant microbiome-metabolome correlation was observed at both time points, however no significant difference was found between the time points.

Figure 4. Forest plots of each prediction performance metric (R-squared - Panel A, Spearman correlation Panel B) for each time point ( 6 weeks $(n=158), 12$ months $(n=282))$ across all 36 metabolites and 4 machine learning models. 95\% credible interval and predictive posterior means were generated using Bayesian modelling of the evaluation statistic (Methods) after 100 repeats of 5-fold nested cross validation. Red vertical lines indicate a value of 0 for the evaluation metric (equivalent to null model). Metabolites were classified as predictable if the null value did not lie within the estimated $95 \%$ credible interval. For most metabolites, predictive performance was not significantly better than null models

Figure 5. Comparative analysis predictive model performance across all metabolites in the targeted dataset for both 6-weeks $(n=158)$ and 12-months $(n=282)$ time points. Top panel shows superimposed boxplots and violin plots of the distribution of predictive posterior mean for each evaluation metric across all 36 metabolites. Bottom panels show aggregated model rankings for all metabolites using R-squared (left) and Spearman correlation (right) using Borda scores (Methods). Higher scores indicate that a model was consistently selected as a better performing. Relatively similar Borda scores and cross-metabolite average predictive performances 
923

924

925

926

927

928

929

930

931

932

933

934

935

936

937

938

939

940

941

942

943

944

945

946

947

indicate that no model was clearly the most performant. However, support vector machines (with radial basis function kernel) was highest scoring model.

Figure S1. Inter-omics Procrustes biplots comparing PCoA ordinations of untargeted metabolite profiles and taxonomic relative abundances for 6 weeks (left panels) $(n=158)$ and 12 months (right panels) $(n=262)$. Top panels present analyses based on ordinations from Euclidean distances of genus level abundances after centered log ratio transformation and Euclidean distances of arcsine square root transformed metabolite relative abundances. Bottom panel presents analyses based on generalized Unifrac distance of amplicon sequence variant (ASV) relative abundances and Euclidean distances of arcsine square root transformed metabolite relative abundances.

Figure S2. Pairwise Spearman correlation of metabolite bins and genus-level taxonomic abundances for 6weeks (panel $\mathrm{A}, \mathrm{N}=168$ ) and 12-months (panel $\mathrm{B}, \mathrm{N}=282$ ) infants. Left panel displays the overall correlation pattern, where non-significant correlations are not colored (false discovery rate (FDR) controlled q-value < 0.05). Right panel displays the same heatmap restricted to taxa and metabolites selected by the sparse CCA procedure. Additionally, correlation coefficient of the first SCCA variate pair, bootstrapped $95 \%$ confidence interval and permutation $\mathrm{p}$-value are also reported.

Figure S3. Comparative analysis predictive model performance across all metabolites in the untargeted dataset for both 6-weeks $(n=158)$ and 12-months $(n=282)$ timepoints. Top panel shows superimposed boxplots and violin plots of the distribution of predictive posterior mean for each evaluation metric across all 208 spectral bins. Bottom panels show aggregated model rankings for all metabolites using R-squared (left) and spearman correlation (right) using Borda scores (Methods).

Figure S4. Results for positive (Panel A) and negative simulations (Panel B). Positive simulations were conducted based on bootstrapped resamples of the original data (12-month time point) and a normally distributed outcome vector which represented a log-transformed metabolite profile. Different levels of model saturation (horizontal, model sparsity (spar) at $0.05,0.1,0.5,0.95$ ) and effect sizes (vertical, signal-to-noise ratio (snr) at $0.5,0.7,3,5)$ were assessed, with 100 data sets generated for each setting combination. 
Negative simulations were conducted based on permutations of the original data (12-month time point), with a total of 1000 permutations. Highly negative outliers were removed for the purposes of visualization

950

951

952

953

954

955

956

957

958

959

960

961

962

963

964

965

966

967

968

969

970

971

972

Figure S5. Inter-omics Procrustes biplots comparing PCoA ordinations of targeted metabolite profiles and taxonomic relative abundances in the sensitivity analyses for 6 weeks (left panels) $(n=65)$ and 12 months (right panels) $(n=65)$. Top panels present analyses based on ordinations from Euclidean distances of genus level abundances after centered log ratio transformation and Euclidean distances of arcsine square root transformed metabolite relative abundances. Bottom panel presents analyses based on generalized Unifrac distance of amplicon sequence variant (ASV) relative abundances and Euclidean distances of arcsine square root transformed metabolite relative abundances

Figure S6. Inter-omics Procrustes biplots comparing PCoA ordinations of untargeted metabolite bin relative concentrations and taxonomic relative abundances in the sensitivity analyses for 6 weeks (left panels) $(n=65)$ and 12 months (right panels) $(n=65)$. Top panels present analyses based on ordinations from Euclidean distances of genus level abundances after centered log ratio transformation and Euclidean distances of arcsine square root transformed metabolite relative abundances. Bottom panel presents analyses based on generalized Unifrac distance of amplicon sequence variant (ASV) relative abundances and Euclidean distances of arcsine square root transformed metabolite relative abundances

Figure S7. Pairwise spearman correlation of concentration-fitted targeted metabolite concentrations and genus-level taxonomic abundances for 6-weeks (panel $A, N=65$ ) and 12-months (panel $B, N=65$ ) infants in sensitivity analyses. Left panel displays the overall correlation pattern, where non-significant correlations are not colored (FDR controlled q-value $<0.05$ ). Right panel displays the same heatmap restricted to taxa and metabolites selected by the SCCA procedure. Additionally, correlation coefficient of the first sCCA variate pair, bootstrapped $95 \%$ confidence interval $(n b o o t=5000)$ and permutation $p$-value $(n p e r m=1000)$ are also reported.

Figure S8. Pairwise spearman correlation of untargeted metabolite bin relative concentrations and genus-level taxonomic abundances for 6-weeks (panel $\mathrm{A}, \mathrm{N}=65$ ) and 12-months (panel $\mathrm{B}, \mathrm{N}=65$ ) infants in sensitivity 
973 analyses. Left panel displays the overall correlation pattern, where non-significant correlations are not colored

974 (FDR controlled q-value < 0.05). Right panel displays the same heatmap restricted to taxa and metabolites

975 selected by the sCCA procedure. Additionally, correlation coefficient of the first sCCA variate pair,

976 bootstrapped $95 \%$ confidence interval $($ nboot $=5000)$ and permutation $p$-value $($ nperm $=1000)$ are also

977 reported

978 Table S1. Metabolites selected for targeted analysis and their potential biological functions. 
Figures

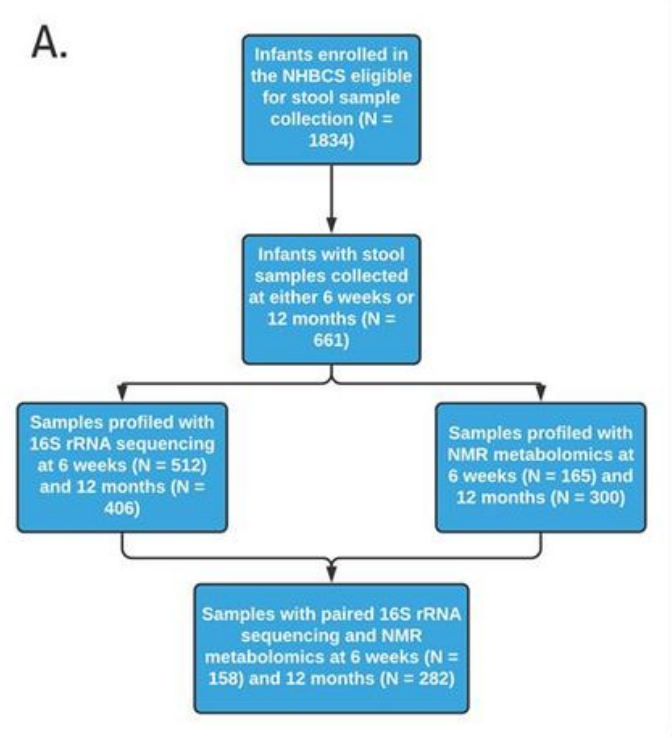

B.

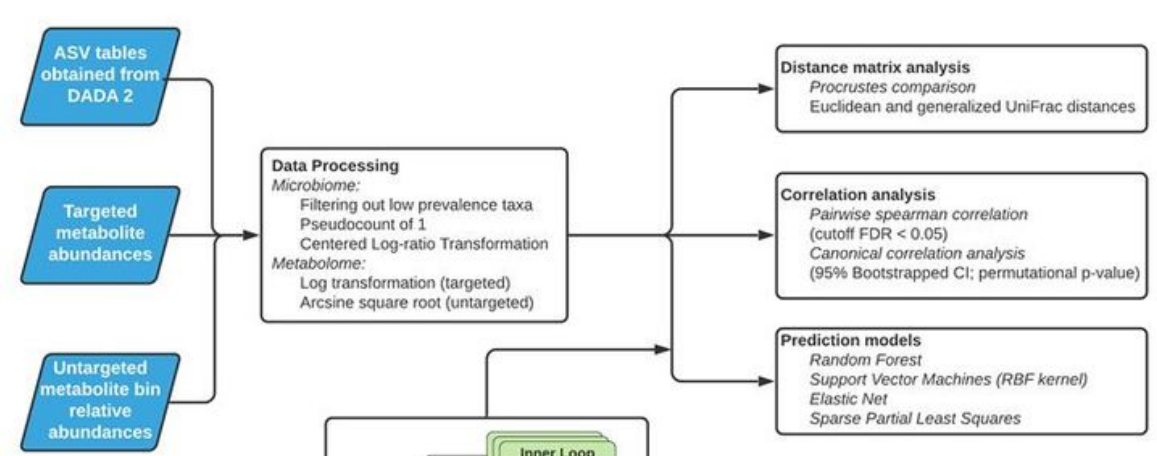

Figure 1

Overview of the analysis. Panel A describes the subject selection workflow and panel B describes the analytic pipeline. 
$\begin{array}{lc}\text { A. Euclidean-Euclidean } & \begin{array}{c}\mathrm{p} \text {-value }=0.057 \\ \text { Sum of Squares: } 0.98\end{array}\end{array}$

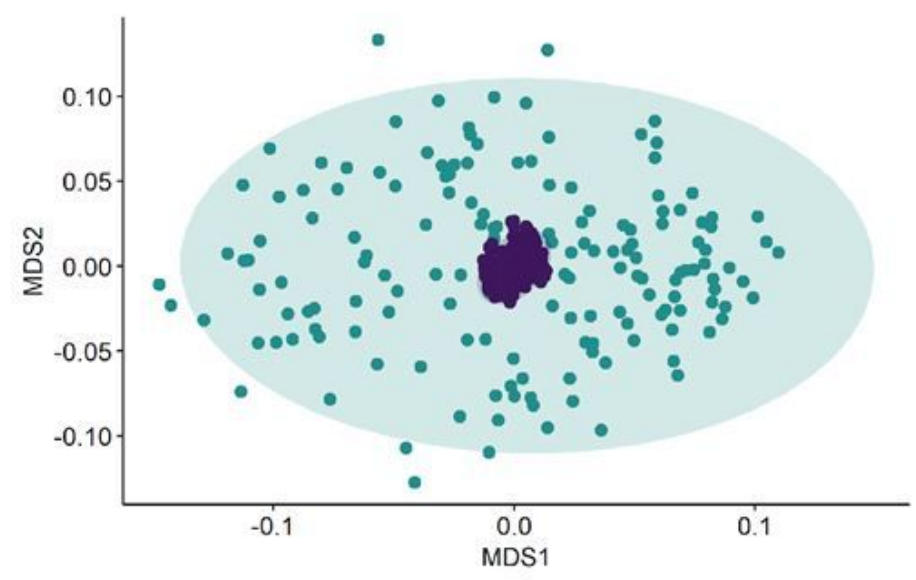

B. Gunifrac-Euclidean

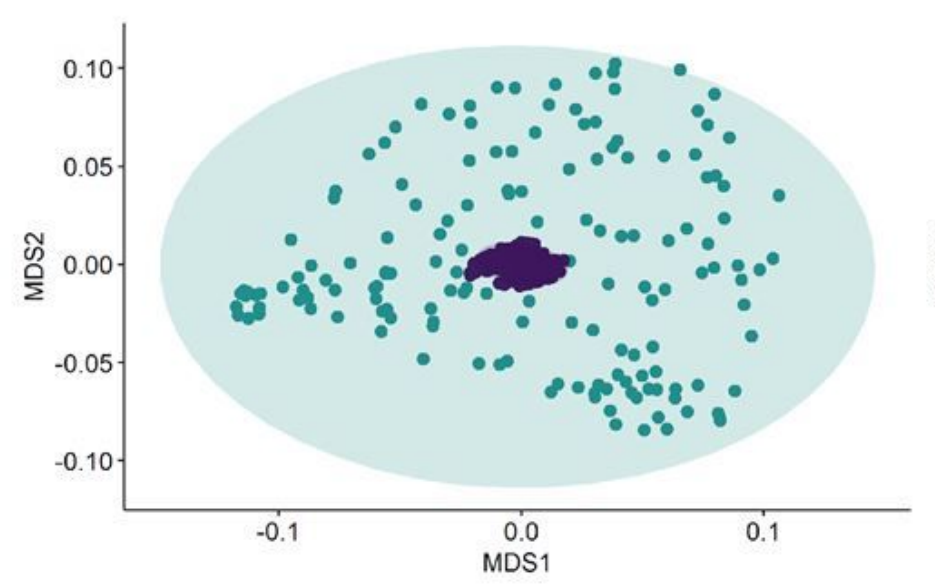

6 weeks
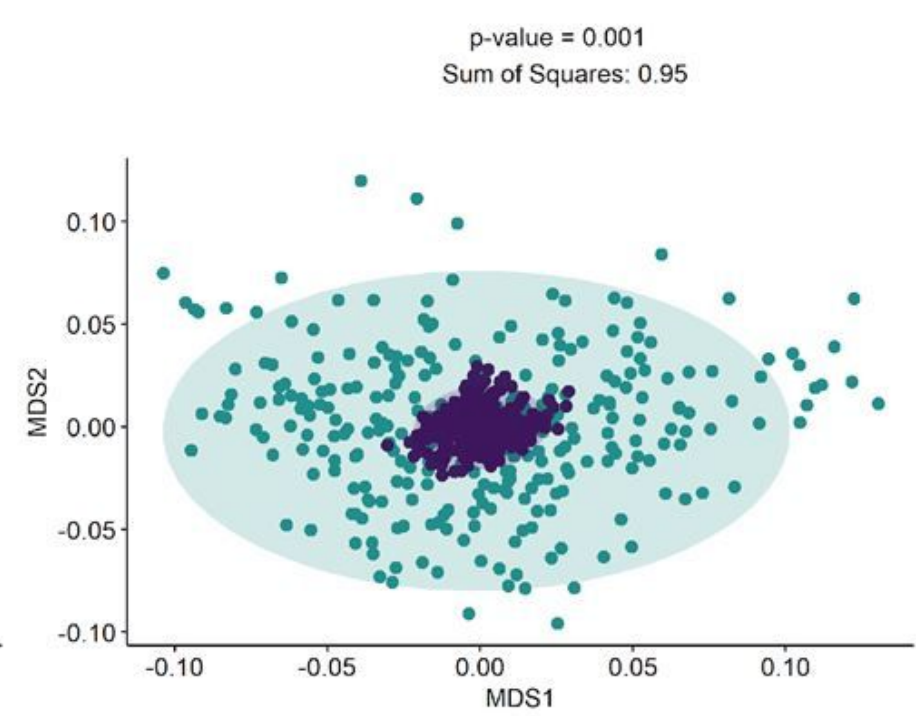

$\mathrm{p}$-value $=0.069$

Sum of Squares: 0.99

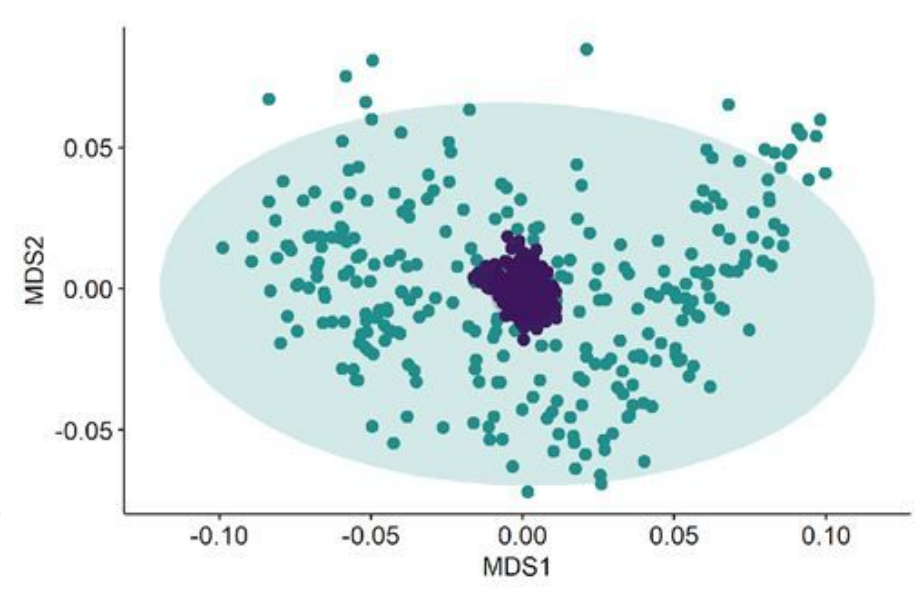

12 months

Ordination • Metabolites • Taxonomy

\section{Figure 2}

Inter-omics Procrustes biplots comparing PCoA ordinations of targeted metabolite profiles and taxonomic relative abundances for 6 weeks (left panels) $(n=158)$ and 12 months (right panels) $(n=262)$. Top panels present analyses based on ordinations from Euclidean distances of genus level abundances after centered log ratio transformation and Euclidean distances of log-transformed metabolite profiles. Bottom panel presents analyses based on gUniFrac distance of amplicon sequence variant (ASV) relative abundances and Euclidean distances of log-transformed metabolite profiles. There were significant associations between the microbiome and the metabolome (both targeted and untargeted) when utilizing Euclidean distances, however this association goes away when the gUniFrac distance was employed for the targeted metabolites only. 

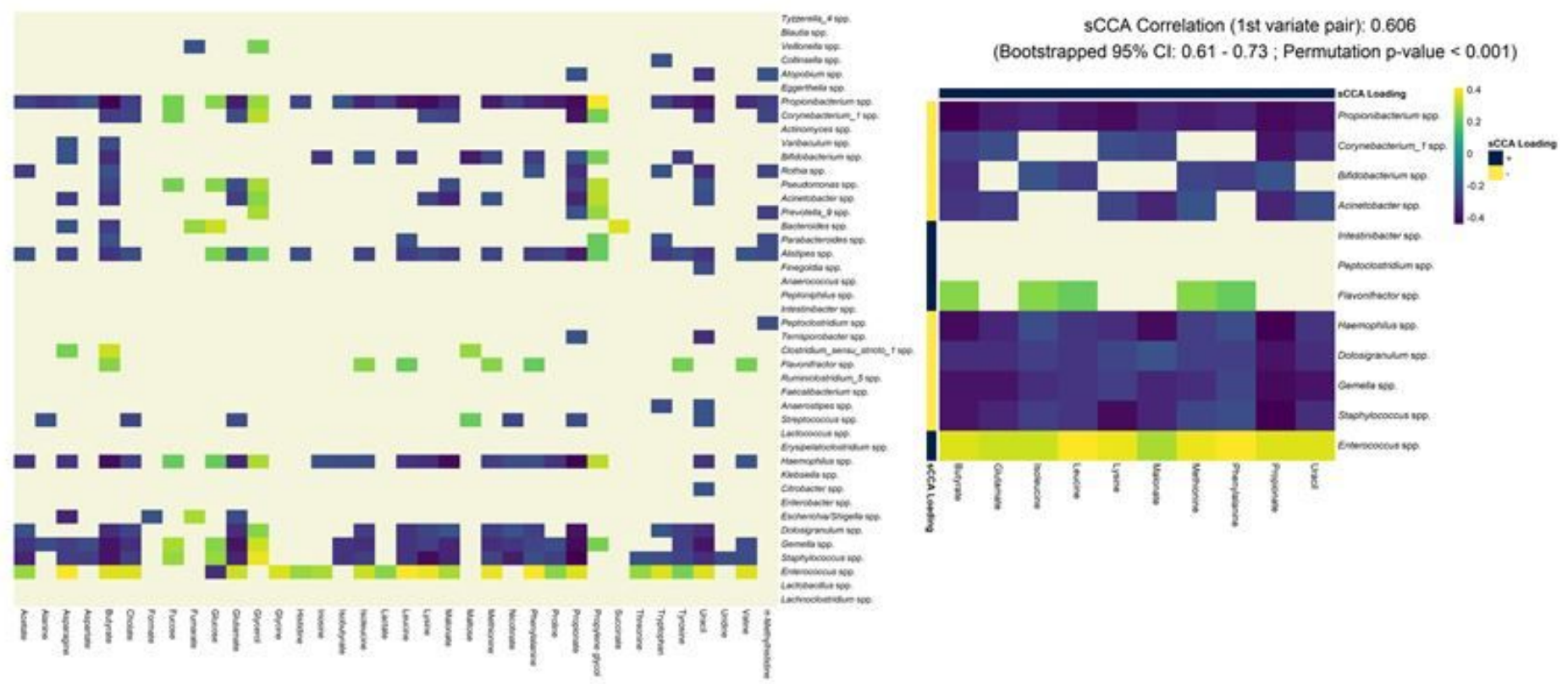

\section{B. 12 Months}
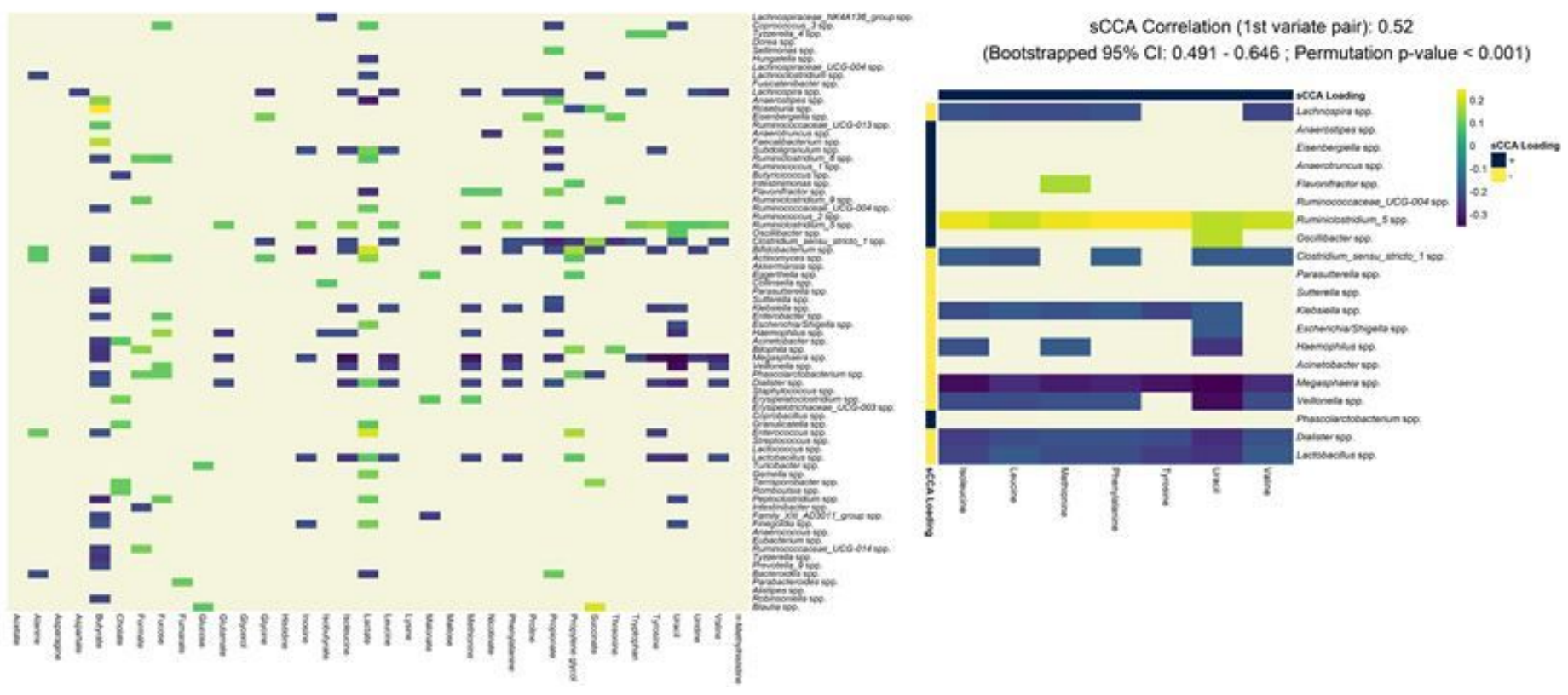

Figure 3

Pairwise Spearman correlation of concentration-fitted metabolites and genus-level taxonomic abundances for 6-weeks (panel $A, N=168$ ) and 12-months (panel $B, N=282$ ) infants. Left panel displays the overall correlation pattern, where non-significant correlations are not colored (false discovery rate (FDR) controlled q-value $<0.05$ ). Right panel displays the same heatmap restricted to taxa and metabolites selected by the sparse CCA procedure. Additionally, correlation coefficient of the first SCCA variate pair, bootstrapped $95 \%$ confidence interval and permutation $p$-value are also reported. Significant microbiome-metabolome correlation was observed at both time points, however no significant difference was found between the time points. 
A. R-squared
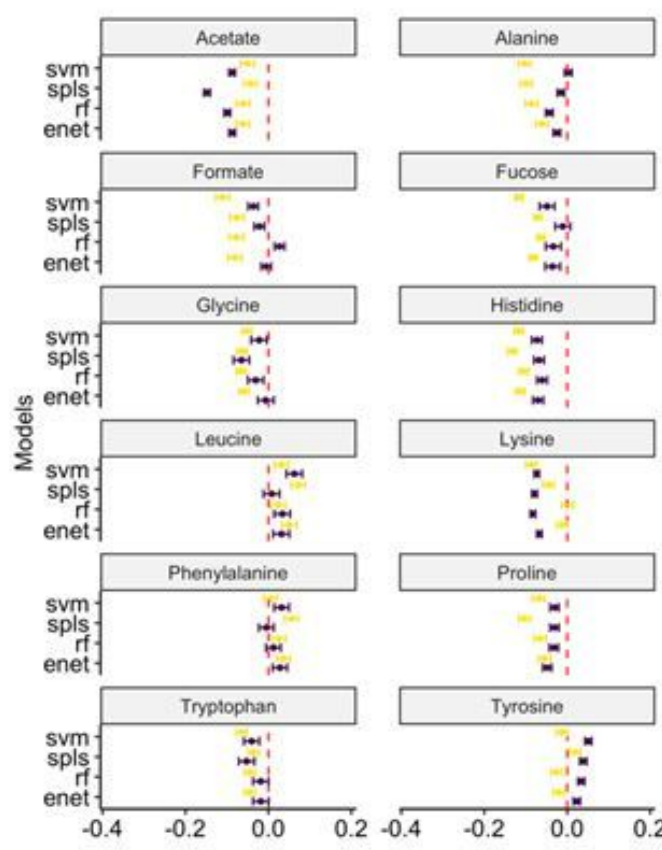

B. Spearman Correlation
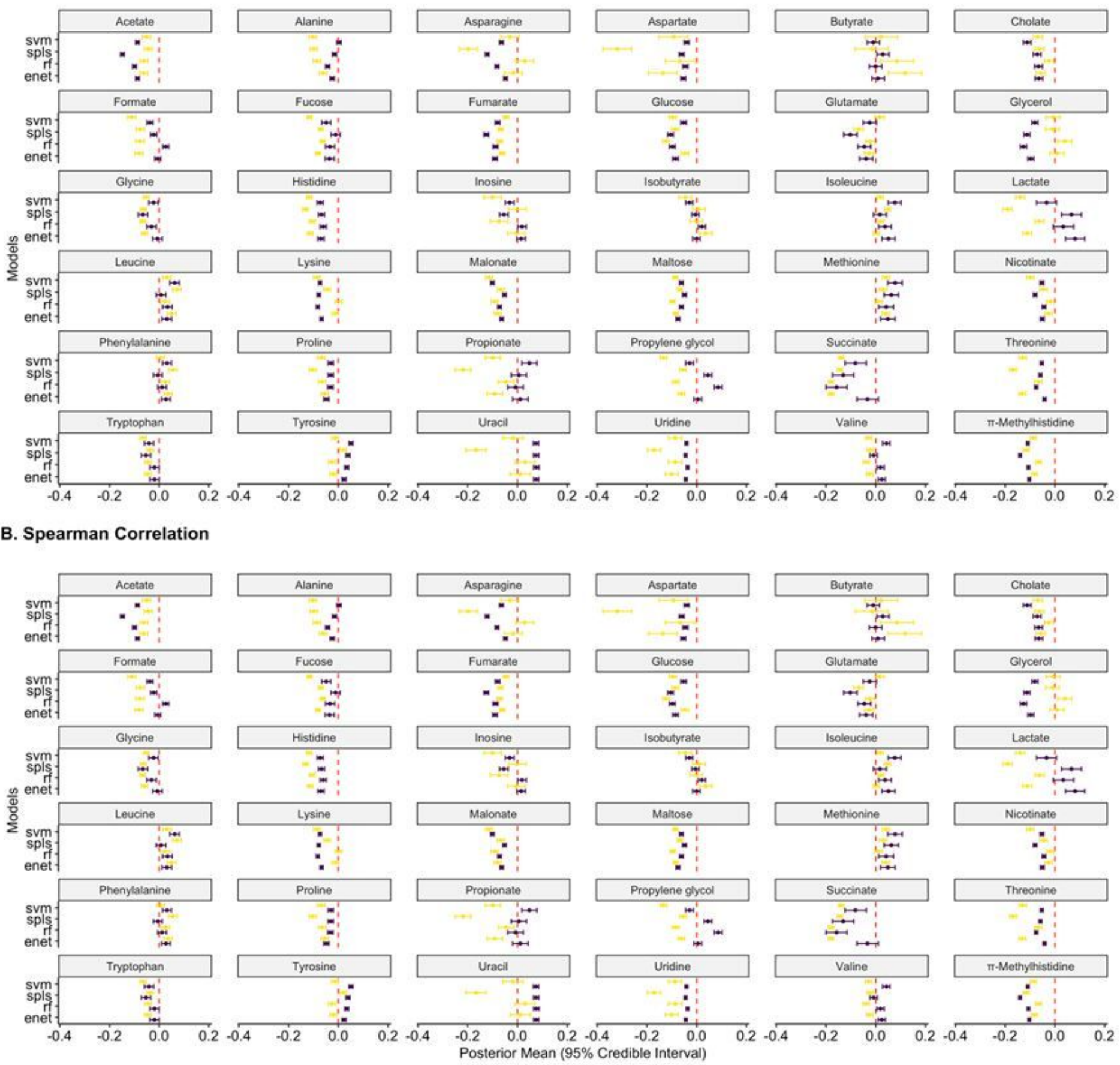

\section{Figure 4}

Forest plots of each prediction performance metric (R-squared - Panel A, Spearman correlation - Panel B) for each time point ( 6 weeks $(n=158), 12$ months $(n=282)$ ) across all 36 metabolites and 4 machine learning models. $95 \%$ credible interval and predictive posterior means were generated using Bayesian modelling of the evaluation statistic (Methods) after 100 repeats of 5 -fold nested cross validation. Red vertical lines indicate a value of 0 for the evaluation metric (equivalent to null model). Metabolites were classified as predictable if the null value did not lie within the estimated $95 \%$ credible interval. For most metabolites, predictive performance was not significantly better than null models 


\section{R-squared}

Time $12 \mathrm{M}$ $6 \mathrm{~W}$
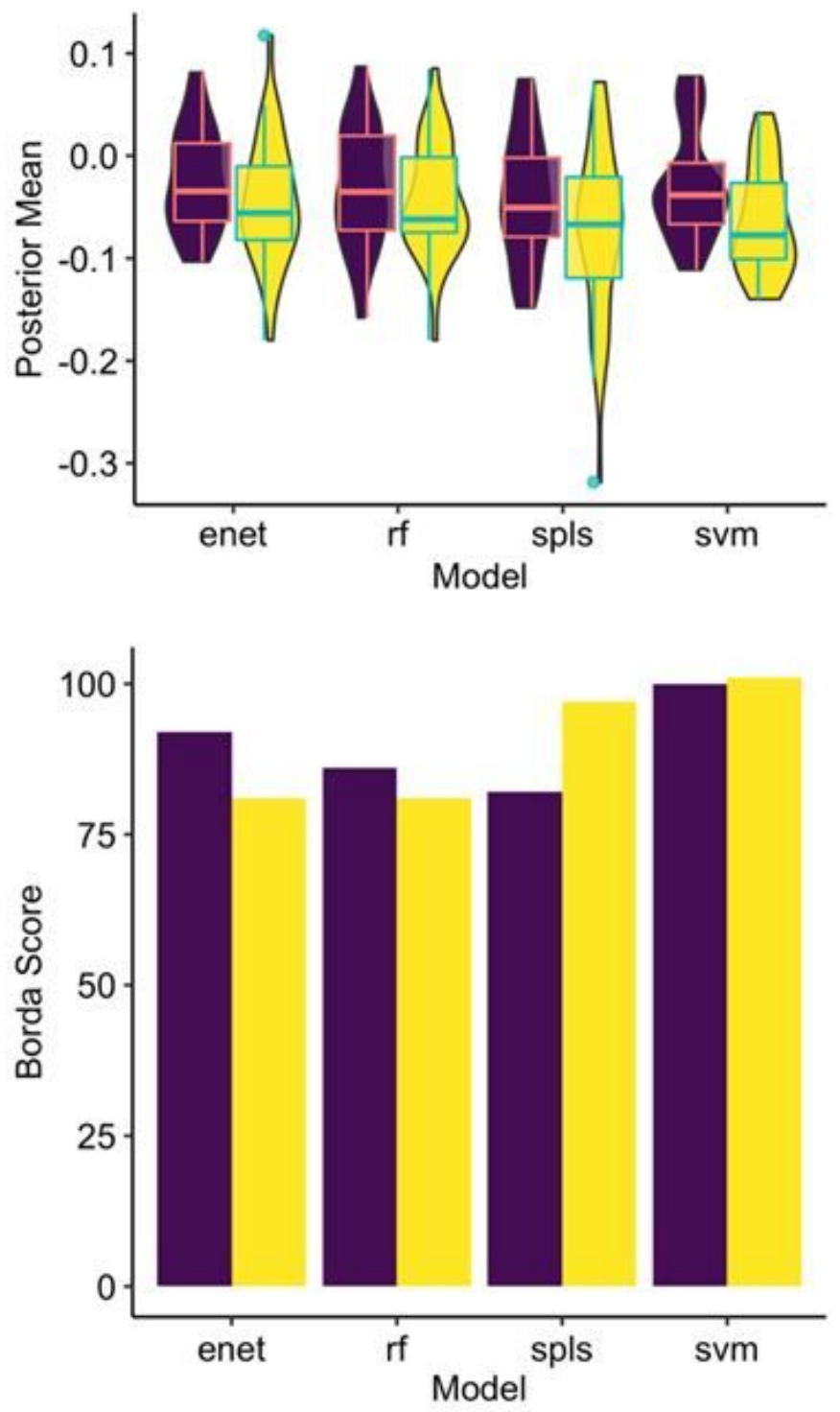

Spearman correlation

Time $12 \mathrm{M}$ $6 \mathrm{~W}$
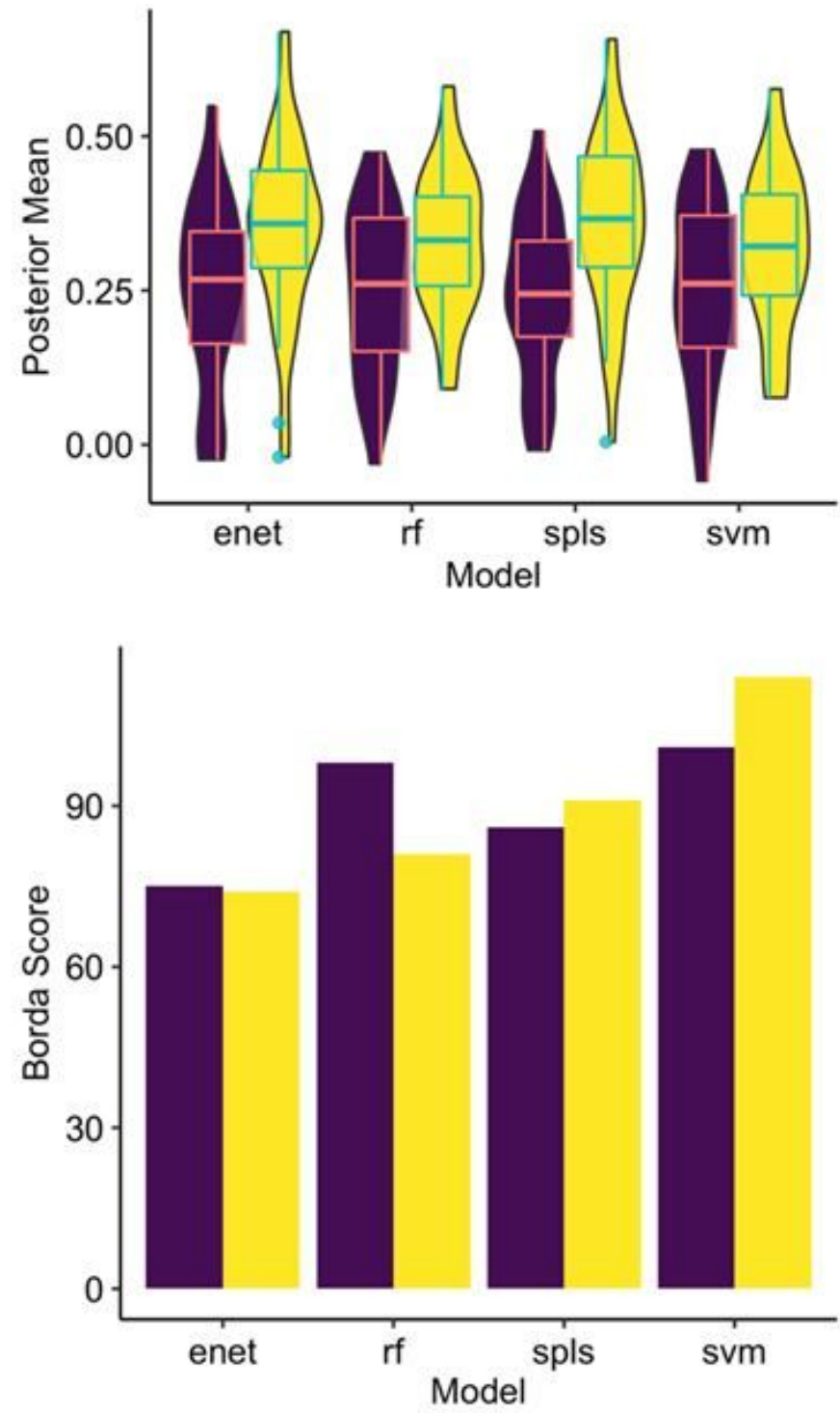

Figure 5

Comparative analysis predictive model performance across all metabolites in the targeted dataset for both 6-weeks $(n=158)$ and 12-months $(n=282)$ time points. Top panel shows superimposed boxplots and violin plots of the distribution of predictive posterior mean for each evaluation metric across all 36 metabolites. Bottom panels show aggregated model rankings for all metabolites using R-squared (left) and Spearman correlation (right) using Borda scores (Methods). Higher scores indicate that a model was consistently selected as a better performing. Relatively similar Borda scores and cross-metabolite average predictive performances indicate that no model was clearly the most performant. However, support vector machines (with radial basis function kernel) was highest scoring model. 


\section{Supplementary Files}

This is a list of supplementary files associated with this preprint. Click to download.

- additionalfile3.xlsx

- additionalfile2.xlsx

- additionalfile1.docx 